\title{
Momentum space parity-odd CFT 3-point functions
}

\author{
Sachin Jain, ${ }^{a}$ Renjan Rajan John, ${ }^{a}$ Abhishek Mehta, ${ }^{a}$ Amin A. Nizami ${ }^{b}$ \\ and Adithya Suresh ${ }^{a}$
}

${ }^{a}$ Indian Institute of Science Education and Research, Homi Bhabha Road, Pashan, Pune 411 008, India

${ }^{b}$ Department of Physics, Ashoka University, Plot No. 2, Rajiv Gandhi Education City, National Capital Region P.O. Rai, Sonepat Haryana-131029, India

E-mail: sachin.jain@iiserpune.ac.in, renjan.john@acads.iiserpune.ac.in, abhishek.mehta@students.iiserpune.ac.in, aan27cam@gmail.com, s.adithya@students.iiserpune.ac.in

ABSTRACT: We study the parity-odd sector of 3-point functions comprising scalar operators and conserved currents in conformal field theories in momentum space. We use momentum space conformal Ward identities as well as spin-raising and weight-shifting operators to fix the form of some of these correlators. Wherever divergences appear we discuss their regularisation and renormalisation using appropriate counter-terms.

Keywords: Conformal and W Symmetry, Conformal Field Theory

ArXiv EPrint: 2101.11635 


\section{Contents}

1 Introduction 1

2 Two approaches to determining momentum space correlators 3

2.1 Using conformal Ward identity: strategy 3

2.2 Dilatation and special conformal Ward identities 4

2.2.1 Divergences 5

$\begin{array}{lll}2.2 .2 & \text { Counter-terms } & 6\end{array}$

$\begin{array}{lll}2.3 & \text { Using weight-shifting and spin-raising operators } & 6\end{array}$

2.3.1 Subtleties with the weight-shifting and spin-raising operator approach 7

3 Allowed parity-odd 3-point functions in various dimensions $\quad 8$

3.1 Three-dimensions 8

3.2 Four-dimensions 8

$\begin{array}{llr}3.3 & \text { Five-dimensions and above } & 9\end{array}$

4 Momentum space parity-odd 3-point functions in three-dimensions using $\begin{array}{lr}\text { conformal Ward identity } & \mathbf{9}\end{array}$

$4.1\left\langle J^{\mu} O O\right\rangle_{\text {odd }} \quad 9$

$4.2\left\langle J^{\mu} J^{\nu} O\right\rangle_{\text {odd }} \quad 10$

$\begin{array}{lll}4.2 .1 & \text { Divergences and renormalization } & 13\end{array}$

$\begin{array}{lll}4.3 & \text { Non-Abelian }\langle J J O\rangle_{\text {odd }} & 14\end{array}$

5 Parity-odd spin-raising \& weight-shifting operators in three-dimensions $\mathbf{1 5}$

$\begin{array}{lll}5.1 & \text { Parity-odd operators } & 15\end{array}$

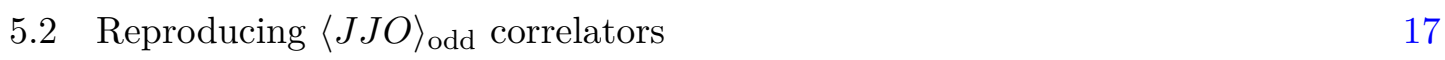

$\begin{array}{lll}5.2 .1 & \left\langle J^{\mu} J^{\nu} O_{2}\right\rangle_{\text {odd }} & 18\end{array}$

$\begin{array}{lll}5.2 .2\left\langle J^{\mu} J^{\nu} O_{4}\right\rangle_{\text {odd }} & 18\end{array}$

$\begin{array}{lll}5.2 .3\left\langle J^{\mu} J^{\nu} O_{5}\right\rangle_{\text {odd }} & 19\end{array}$

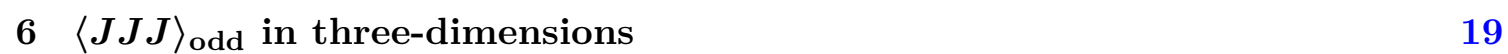

$7\langle\boldsymbol{T T O}\rangle_{\text {odd }}$ in three-dimensions $\quad 21$

$\begin{array}{lll}7.1 & \left\langle\mathrm{TTO}_{2}\right\rangle \text { for free fermionic theory } & 22\end{array}$

$7.2\left\langle\mathrm{TTO}_{2}\right\rangle_{\text {odd }}$ using parity-odd spin-raising and weight-shifting operators $\quad 24$

$\left.7.3\langle\text { TTO }\rangle_{1}\right\rangle_{\text {odd }}$ using parity-odd spin-raising and weight-shifting operators $\quad 24$

8 Momentum space parity-odd 3-point correlators in four-dimensions $\quad \mathbf{2 5}$

$\begin{array}{lll}8.1 & \text { Spin-raising and weight-shifting operators } & 25\end{array}$

$\begin{array}{lll}8.2\langle J J\rangle_{\text {odd }} & 26\end{array}$

$\begin{array}{lll}9 & \text { Discussion } & 27\end{array}$ 
B Parity-odd two-point functions $\quad 29$

$\begin{array}{lll}\text { B.1 Four and higher dimensions } & 29\end{array}$

$\begin{array}{lll}\text { B.2 Three-dimensions } & 29\end{array}$

$\begin{array}{ll}\text { C Schouten identities } & 30\end{array}$

D Details of $\left\langle\mathrm{TTO}_{\mathbf{2}}\right\rangle$ computation $\quad 31$

D.1 Transverse and trace Ward identities 32

D.2 Details of longitudinal part 33

E Parity-even spin-raising and weight-shifting operators 33

F Embedding space parity-odd correlation functions in four-dimensions $\quad 34$

$\begin{array}{lll}\text { F.1 }\langle J T\rangle_{\text {odd }} & 34\end{array}$

$\begin{array}{ll}\text { F.2 }\langle T T T\rangle_{\text {odd }} & 35\end{array}$

\section{Introduction}

Conformal Field Theories (CFTs) have wide applicability in diverse areas of physics, and are central to our understanding of quantum field theory in terms of RG flows. While CFTs are well studied in position space and Mellin space, they are relatively less studied in momentum space. Recent works on aspects of momentum space CFTs include [1-38]. CFTs in momentum space find applications in cosmology [39-48], condensed matter physics [49, 50], study of anomalies [51-55], Hamiltonian truncation methods for strongly coupled field theories [56, 57] and, of course, the conformal bootstrap program [17, 21, 26, 58].

From the perspective of perturbative field theory which is naturally formulated in momentum space, it is of interest to study CFTs in the same setting. Flat space scattering amplitudes are, via AdS/CFT, directly related to the flat space limit of CFT correlators in momentum space [59]. ${ }^{1}$ Studying momentum space CFT correlators can therefore shed light on the structure of flat space amplitudes. Interestingly, evidence for the double copy structure - which exists for flat space amplitudes — was seen directly in momentum space CFT 3-point correlators in [16, 27]. An important simplification in momentum space is that 4-point conformal blocks can be constructed from 3-point functions in a relatively straightforward manner in momentum space [26, 37]. Another significant application is in the cosmological setting where the CMB bispectrum which is a measure of non-gaussianity is given by the 3 -point function in momentum space.

Three-point functions of scalar and spinning operators have a simple, well-known form in position space - this is most easily seen by going to the embedding space. However,

\footnotetext{
${ }^{1}$ There are analogous, though somewhat less straightforward relations in Mellin space [60, 61] and position space [62-64].
} 
their momentum space analogues are quite complicated. For example, the scalar 3-point correlator in momentum space is solved in terms of triple- $K$ integrals which can involve divergences [2]. A careful treatment would require the regularisation and renormalisation of these divergences $[2,4] .^{2}$ The story of spinning correlators is even more complicated $[2$, $3,9,12]$.

Parity odd structures in CFTs have been investigated in [65-70]. However, parity-odd correlation functions in momentum space have received very little attention. In threedimensions they appear naturally. Consider, for example, the free fermion theory in threedimensions. The scalar primary operator with the lowest dimension is given by $\bar{\psi} \psi$, and it is odd under parity. A correlator with an odd number of insertions of this operator will be parity-odd. Another place where parity-odd structures arise naturally is CFTs with a broken parity. A prime example of such CFTs is Chern-Simons matter theories [71-80]. In [73], it was argued that in such theories:

$$
\left\langle J_{s_{1}} J_{s_{2}} J_{s_{3}}\right\rangle=\alpha\left\langle J_{s_{1}} J_{s_{2}} J_{s_{3}}\right\rangle_{\text {Free-Boson }}+\beta\left\langle J_{s_{1}} J_{s_{2}} J_{s_{3}}\right\rangle_{\text {Free-Fermion }}+\gamma\left\langle J_{s_{1}} J_{s_{2}} J_{s_{3}}\right\rangle_{\text {odd }}
$$

where $J_{s}$ is the spin $s$ conserved current, the subscript odd indicates a parity-odd contribution to the correlator and $\alpha, \beta, \gamma$ are theory dependent constants. In three-dimensions ${ }^{3}$ one cannot obtain the parity-odd part of generic three-point correlators of spinning operators from the free theory and one needs to use CFT techniques. ${ }^{4}$ There are other instances where parity-odd contribution might be interesting. For example, although parity-violating correlations are as yet unobserved, the CMB bispectrum - a measure of non-gaussianity - could contain parity-odd contributions. Such a contribution can potentially arise, for example, from an inflationary action with higher derivative corrections such as cubic Weyl tensor terms [39]. ${ }^{5}$ It may seem that such terms could source contributions to the primordial graviton bispectrum, however in [82] it was shown that the parity-violating (odd) contribution in the non-gaussianity of the primordial gravitational waves CMB vanishes in an exact de-Sitter background, but exists in inflationary quasi de-Sitter where it is proportional to the slow-roll parameter. Other studies of parity-violating CMB bispectrum include [83-85].

In this paper, we use two complementary approaches to determine the momentum space structure of parity-odd CFT 3-point functions. The first method is the more direct one and involves solving momentum-space Ward identities. This approach was developed and used for the parity-even sector in [2].

The second approach utilises spin-raising and weight-shifting operators. These have been used in the conformal bootstrap literature $[66,67,86]$ and more recently in fixing

\footnotetext{
${ }^{2}$ In position space one could get rid of divergences by working at non-coincident points, but in momentum space one cannot do this and this leads to UV divergences.

${ }^{3}$ In four dimensions, the 3-point function of stress-tensor is given by [81]$$
\langle T T T\rangle=a\langle T T T\rangle_{\mathrm{FB}}+b\langle T T T\rangle_{\mathrm{FF}}+c\langle T T T\rangle_{\mathrm{Maxwell}} .
$$

${ }^{4}$ For specific correlators such as $\left\langle J_{s_{1}} J_{s_{2}} O_{2}\right\rangle$ one can obtain the parity-odd part from the free fermion theory. In specific kinematic regimes certain parity-odd correlation functions have been computed using Feynman diagram techniques in Chern-Simons matter theories [74, 75, 79].

${ }^{5}$ These are of the form $\int \widetilde{W} W^{2}$ where $W$ denotes the Weyl tensor and $\widetilde{W}$ its Hodge-dual.
} 
the form of cosmological correlators [47, 48]. We will construct parity-odd spin-raising and weight-shifting operators in momentum space and use them on scalar seed correlators to generate parity-odd spinning correlators.

The rest of this paper is organised as follows. In section 2, besides setting up the notation and terminology, we outline the two different techniques that we use in this paper to determine parity-odd 3-point functions. We also briefly discuss the divergences that arise, and their regularisation. In section 3, we give an overview of the possible parity-odd 3point structures in embedding space in various dimensions. In section 4 , we use momentum space conformal Ward identities to fix the parity-odd part of $\langle J J O\rangle$. In section 5 , we construct parity-odd spin-raising and weight-shifting operators in momentum space and use them to determine spinning correlators by their action on simple scalar seed correlators. We show that the results for $\langle J J O\rangle$ obtained using spin-raising and weight-shifting operators match the results for the same obtained in section 4 . In section 6 , we compute the parityodd 3-point function of the spin-one conserved current using weight-shifting and spinraising operators. In section 7 , we compute the form of the odd part of $\left\langle T T O_{\Delta}\right\rangle$ for chosen conformal dimensions of $O$. In the free fermion theory, where $\Delta=2$, we match the results obtained using spin-raising and weight-shifting operators with the answer obtained by an explicit computation in the free theory. In section 8 , we construct parity-odd spin-raising and weight-shifting operators in four-dimensions and use them to construct the parity-odd part of the non-trivial correlator $\langle J J J\rangle$ in four dimensions. We conclude with a discussion in section 9. In the appendices we elaborate on various technical details. In appendix A, we review essential details on the embedding space formalism. In appendix B, we discuss the basics of momentum space two-point functions. In appendix C, we present the Schouten identities relevant to us. In appendix D, we give some computational details of the results presented in section 7. Appendix E gives the form of various parity-even spin-raising and weight-shifting operators. In appendix F, we argue on grounds of permutation symmetry that certain 3-point correlators with spinning operators in four-dimensions vanish.

\section{Two approaches to determining momentum space correlators}

Determining correlation functions is a significantly harder task in momentum space than in position space. For parity-odd correlators this gets even more tedious. We will now discuss two different approaches to determining momentum space correlators. We also discuss certain subtleties and limitations associated with the two approaches.

\subsection{Using conformal Ward identity: strategy}

In the first approach, following $[1,2]$ and $[4,9,10,12,14]$ where parity-even 3 -point functions were determined, we start with an ansatz of the form $\sum_{m} A_{m}\left(k_{i}\right) \mathcal{T}_{m}$ for the correlator. Here $\mathcal{T}_{m}$ are all possible tensor structures that are allowed by symmetry and $A_{m}$ are form factors which are functions of the momenta magnitudes $\left(k_{i}\right)$. The form factors are constrained by permutation symmetries (if any) of the correlator and by momentum space Ward identities. The latter lead to partial differential equations which can then be solved to determine the form factors, up to undetermined constants that depend on the 
specific theory. In section 4, we use this method to fix the parity-odd part of certain 3-point functions in momentum space. An excellent mathematica package that we found useful in these computations is [87].

Let us now describe the momentum space Ward identities associated with dilatation symmetry and special conformal transformations.

\subsection{Dilatation and special conformal Ward identities}

We will denote the $n$-point Euclidean correlation function of primary operators $\mathcal{O}_{1}, \ldots, \mathcal{O}_{n}$ by $\left\langle\mathcal{O}_{1}\left(\boldsymbol{k}_{1}\right) \ldots \mathcal{O}_{n}\left(\boldsymbol{k}_{n}\right)\right\rangle$. We suppress the Lorentz indices of the operators for brevity. The correlator with the momentum conserving delta function stripped off is denoted as:

$$
\left\langle\mathcal{O}_{1}\left(\boldsymbol{k}_{1}\right) \ldots \mathcal{O}_{n}\left(\boldsymbol{k}_{n}\right)\right\rangle \equiv(2 \pi)^{d} \delta^{(3)}\left(\boldsymbol{k}_{1}+\ldots+\boldsymbol{k}_{n}\right)\left\langle\left\langle\mathcal{O}_{1}\left(\boldsymbol{k}_{1}\right) \ldots \mathcal{O}_{n}\left(\boldsymbol{k}_{n}\right)\right\rangle\right\rangle
$$

An $n$-point correlator with scalar or spinning operator insertions satisfies the following dilatation Ward identity [2]:

$$
0=\left[-(n-1) d+\sum_{j=1}^{n} \Delta_{j}-\sum_{j=1}^{n-1} k_{j}^{\alpha} \frac{\partial}{\partial k_{j}^{\alpha}}\right]\left\langle\left\langle\mathcal{O}_{1}\left(\boldsymbol{k}_{1}\right) \ldots \mathcal{O}_{n}\left(\boldsymbol{k}_{n}\right)\right\rangle\right\rangle .
$$

This constrains the correlator to have the following scaling behaviour:

$$
\left\langle\left\langle\mathcal{O}_{1}\left(\lambda \boldsymbol{k}_{1}\right) \ldots \mathcal{O}_{n}\left(\lambda \boldsymbol{k}_{n}\right)\right\rangle\right\rangle=\lambda^{-\left[(n-1) d-\sum_{i=1}^{n} \Delta_{i}\right]}\left\langle\left\langle\mathcal{O}_{1}\left(\boldsymbol{k}_{1}\right) \ldots \mathcal{O}_{n}\left(\boldsymbol{k}_{n}\right)\right\rangle\right\rangle .
$$

The special conformal Ward identity on an $n$-point correlator with both scalar and spinning operators is [2]:

$$
\begin{aligned}
0= & \sum_{j=1}^{n-1}\left[2\left(\Delta_{j}-d\right) \frac{\partial}{\partial k_{j}^{\kappa}}-2 k_{j}^{\alpha} \frac{\partial}{\partial k_{j}^{\alpha}} \frac{\partial}{\partial k_{j}^{\kappa}}+k_{j}^{\kappa} \frac{\partial}{\partial k_{j}^{\alpha}} \frac{\partial}{\partial k_{j \alpha}}\right]\left\langle\left\langle\mathcal{O}_{1}\left(\boldsymbol{k}_{1}\right) \ldots \mathcal{O}_{n}\left(\boldsymbol{k}_{n}\right)\right\rangle\right\rangle \\
& +2 \sum_{j=1}^{n-1} \sum_{k=1}^{n_{j}}\left(\delta^{\mu_{j k} \kappa} \frac{\partial}{\partial k_{j}^{\alpha_{j k}}}-\delta_{\alpha_{j k}}^{\kappa} \frac{\partial}{\partial k_{j_{\mu_{j k}}}}\right) \\
& \times\left\langle\left\langle\mathcal{O}_{1}^{\mu_{11} \ldots \mu_{1 r_{1}}}\left(\boldsymbol{k}_{1}\right) \ldots \mathcal{O}_{j}^{\mu_{j 1} \ldots \alpha_{j k} \ldots \mu_{j r_{j}}}\left(\boldsymbol{k}_{j}\right) \ldots \mathcal{O}_{n}^{\mu_{n 1} \ldots \mu_{n r_{n}}}\left(\boldsymbol{k}_{n}\right)\right\rangle\right\rangle
\end{aligned}
$$

In the second line of the r.h.s. of the above equation, the indices of the generator mix with the spin indices of the correlator. In principle, one can solve this equation and get the desired correlator [2]. However, for parity-odd structures in three-dimensions, the computation gets complicated and has not yet been done.

We will always be working with correlation functions with the momentum conserving delta function stripped off. From here on we will drop the double angular brackets notation to avoid clutter and use single angular brackets everywhere.

We will also use the terminology of primary and secondary conformal Ward identities [2]. A 3-point momentum space correlator can be expanded as $\sum A_{i} \mathcal{T}_{i}$ where the $A_{i}$ are the (scalar) form factors, whereas $\mathcal{T}_{i}$ give a basis for tensor structures. When one considers the action of the special conformal generator $K^{\kappa}$ on this correlators, it naturally results in PDEs for the form factors. 
In brief, a primary Ward identity is a second order PDE for the form factor arising from terms containing $k_{1}^{\kappa}, k_{2}^{\kappa}$ in the conformal Ward identity $K^{\kappa}\langle\ldots\rangle=0$. The remaining PDEs are secondary Ward identities and are first order. See section 5 of [2] for further details and properties.

\subsubsection{Divergences}

Triple- $K$ integrals arise as solutions to primary conformal Ward identities which are second order differential equations [2]. Along with the three momenta, they are expressed in terms of four other parameters:

$$
I_{\alpha\left\{\beta_{1} \beta_{2} \beta_{3}\right\}}\left(k_{1}, k_{2}, k_{3}\right) \equiv \int_{0}^{\infty} d x x^{\alpha} \prod_{j=1}^{3} k_{j}^{\beta_{j}} K_{\beta_{j}}\left(k_{j} x\right)
$$

where $K_{\beta_{j}}$ is a modified Bessel function of the second kind. While the integral is well behaved at its upper limit, it is convergent at $x=0$ only if $[2,9]$ :

$$
\alpha+1-\left|\beta_{1}\right|-\left|\beta_{2}\right|-\left|\beta_{3}\right|>0
$$

When the integral is divergent one can regulate it using two parameters $u$ and $v[2,9]$ :

$$
I_{\alpha\left\{\beta_{1} \beta_{2} \beta_{3}\right\}} \rightarrow I_{\alpha+u \epsilon\left\{\beta_{1}+v \epsilon, \beta_{2}+v \epsilon, \beta_{3}+v \epsilon\right\}}
$$

The regularised triple- $K$ integral is convergent except when $[2,9]$ :

$$
\alpha+1 \pm \beta_{1} \pm \beta_{2} \pm \beta_{3}=-2 n, \quad n \in \mathbb{Z}_{\geq 0}
$$

for any choice of signs. When (2.8) is satisfied, the integral is singular in the regulator $\epsilon$ and we will denote the divergence by the choice of signs $( \pm \pm \pm$ ) for which (2.8) is satisfied. Divergences of the type (- - ) are called ultra-local and they occur when all the three operators are co-incident in position space. In momentum space, this manifests as the divergent term being analytic in all three momenta squared. Such divergences must, in general, be removed using counter-terms that are cubic in the sources, and they give rise to conformal anomalies.

Divergences of the type $(--+)$ and its permutations are called semi-local divergences. In position space, this is a divergence that occurs when two of the operators in the correlator are at co-incident points. In momentum space, the divergence is said to be semi-local when the $O(1 / \epsilon)$ term is analytic in any two of the three momenta squared. In general, these divergences must be removed by counter-terms that have two sources and an operator. Such terms lead to non-trivial beta functions.

Divergences of the kind $(+++)$ and $(++-)$ are non-local and they occur even when all three operators are at separated points in position space. In momentum space, such a divergence is analytic in at most one of the momenta squared. This is not a physical divergence and arises because the triple- $K$ integral representation of the correlator is singular. In this case no counter-term exists and the divergence is removed by imposing the condition that the constant multiplying the triple- $K$ integral vanishes as an appropriate power of $\epsilon$. 


\subsubsection{Counter-terms}

As we discussed above, divergences of the kind $(--+)$ and $(---)$ that correspond to ultra-local and semi-local divergences are removed using suitable counter-terms. In the case of parity-even correlators this has been extensively studied in $[2,4,9,12]$.

We will now list a few potential counter-terms that could turn out to be useful in our study of parity-odd correlators. For ultra-local divergences, for example we have:

$$
\int d^{3} x F_{3}(A) \square^{n} \phi, \quad \int d^{3} x C_{\mu \nu} R^{\mu \nu} \square^{n} \phi, \quad \int d^{3} x C_{\mu \nu} R \nabla^{\mu} \nabla^{\nu} \square^{n} \phi
$$

and for semi-local divergences:

$$
\int d^{3} x \epsilon^{\mu \nu \lambda} F_{\mu \nu} J_{\lambda} \square^{n} \phi, \quad \int d^{3} x A^{\mu} J_{\mu} \square^{n} \phi, \quad \int d^{3} x F^{\mu \nu} J_{\mu} D_{\nu} \phi, \quad \int d^{3} x C_{\mu \nu} T^{\mu \nu} \square^{n} \phi
$$

where $F_{3}(A)$ is the Chern-Simons form in three-dimensions given by,

$$
F_{3}(A)=\epsilon_{\mu \nu \lambda}\left(A_{a}^{\mu} \partial^{\nu} A_{a}^{\lambda}+\frac{2}{3} f^{a b c} A_{a}^{\mu} A_{b}^{\nu} A_{c}^{\lambda}\right),
$$

$C_{\mu \nu}$ is the Cotton-York tensor given by,

$$
C_{\mu \nu}=\nabla^{\rho}\left(R_{\mu}^{\sigma}-\frac{1}{4} R g_{\mu}^{\sigma}\right) \epsilon_{\rho \sigma \nu},
$$

and $R_{\mu \nu}$ and $R$ are the Ricci tensor and the Ricci scalar respectively.

In the above list of possible counter-terms (2.10) we have included certain parity-even terms such as $\int d^{3} x A^{\mu} J_{\mu} \square^{n} \phi$ and $\int d^{3} x F^{\mu \nu} J_{\mu} D_{\nu} \phi$. These counter-terms could give rise to the 2-point function of currents, which has a parity-odd contribution $\left\langle J^{\mu}(p) J^{\nu}(-p)\right\rangle \propto$ $\epsilon^{\mu \nu \rho} p_{\rho}$.

\subsection{Using weight-shifting and spin-raising operators}

The second method of computing correlation functions in momentum space hinges on the technique of weight-shifting and spin-raising operators. In position space, this technique was initiated in [67] and extensively developed in [86]. In this approach, starting from certain seed correlators, the action of conformally covariant weight-shifting and spin-raising operators generates the desired correlator.

To describe this method in some detail, let us consider a spinning correlator $\left\langle J_{s_{1}} J_{s_{2}} J_{s_{3}}\right\rangle$. The first step is to count the number of independent tensor structures associated with this correlator. For parity-even correlators this number in position space is given by [66]:

$$
N_{3 d}^{+}\left(l_{1}, l_{2}, l_{3}\right)=2 l_{1} l_{2}+l_{1}+l_{2}+1-\frac{p(p+1)}{2}
$$

where $p=\max \left(0, l_{1}+l_{2}-l_{3}\right)$. The second step is to consider a seed correlator of the form $\left\langle O_{\Delta_{1}} O_{\Delta_{2}} J_{s_{3}}\right\rangle$ and find out $N_{3 d}^{+}\left(l_{1}, l_{2}, l_{3}\right)$ ways to reach $\left\langle J_{s_{1}} J_{s_{2}} J_{s_{3}}\right\rangle$. This involves acting upon the seed correlator with various spin-raising and weight-shifting operators. In momentum space, we are constrained in our choice of seed correlators because correlators of the form 
$\left\langle J^{(l)} O_{\Delta_{1}} O_{\Delta_{2}}\right\rangle$, where $J^{(l)}$ is a spin- $l$ conserved current, are non-zero only when $\Delta_{1}=\Delta_{2}$. A more convenient approach was recently advocated in $[47,48]$ to compute (parity-even) spinning cosmological correlators where instead of starting from the seed $\left\langle O_{\Delta_{1}} O_{\Delta_{2}} J_{s_{3}}\right\rangle$, one starts from $\left\langle O_{\Delta_{1}} O_{\Delta_{2}} O_{\Delta_{3}}\right\rangle$, and apply spin-raising and weight-shifting operators such that the resulting correlator satisfies the Ward-Takahashi identity. See section 4.2.2 of [48] for an example.

\subsubsection{Subtleties with the weight-shifting and spin-raising operator approach}

In momentum space, one must consider the types of divergences in the seed and target correlators. It is not always possible to reach a target correlator starting from a seed correlator although a naive application of the spin-raising and weight-shifting operators might suggest so. This is most easily understood in the case of scalar correlators. As a concrete example of such a situation, consider the following two correlators in three-dimensions:

$$
\begin{aligned}
\left\langle O_{1}\left(k_{1}\right) O_{1}\left(k_{2}\right) O_{2}\left(k_{3}\right)\right\rangle & =\frac{1}{k_{1} k_{2}} \\
\left\langle O_{2}\left(k_{1}\right) O_{2}\left(k_{2}\right) O_{2}\left(k_{3}\right)\right\rangle & =-\log \left(\frac{k_{1}+k_{2}+k_{3}}{\mu}\right)
\end{aligned}
$$

where $\mu$ is the renormalisation scale. Although it might seem like we can use the weightshifting operator $W_{12}^{++}$(defined in (E.4)) to go from the first correlator to the second, this is clearly not possible as $\left\langle O_{2}\left(k_{1}\right) O_{2}\left(k_{2}\right) O_{2}\left(k_{3}\right)\right\rangle$ violates scale invariance whereas the seed correlator $\left\langle O_{1}\left(k_{1}\right) O_{1}\left(k_{2}\right) O_{2}\left(k_{3}\right)\right\rangle$ does not, i.e.

$$
W_{12}^{++}\left\langle O_{1}\left(k_{1}\right) O_{1}\left(k_{2}\right) O_{2}\left(k_{3}\right)\right\rangle \neq\left\langle O_{2}\left(k_{1}\right) O_{2}\left(k_{2}\right) O_{2}\left(k_{3}\right)\right\rangle
$$

The above example tells us that weight-shifting operators fail to reproduce the correct correlators when the divergence type changes from non-local to semi-local or ultra-local. The conditions for various types of divergences, in terms of scaling dimensions of the operator insertions, are given by:

$$
\begin{array}{|c|c|}
\hline(---) & \Delta_{1}+\Delta_{2}+\Delta_{3}=2 d+2 k_{1} \\
\hline(--+) & \Delta_{1}+\Delta_{2}-\Delta_{3}=d+2 k_{2} \\
\hline(++-) & -\Delta_{1}-\Delta_{2}+\Delta_{3}=2 k_{3} \\
\hline(+++) & \Delta_{1}+\Delta_{2}+\Delta_{3}=d-2 k_{4} \\
\hline
\end{array}
$$

where $k_{1}, k_{2}, k_{3}, k_{4} \geq 0$. We can see that the only time the divergence structure changes is when $k_{i}=0$. For the non-local cases in three-dimensions, these correspond to the following for the seed correlator:

$$
\begin{array}{ll}
\Delta_{3}=\Delta_{1}+\Delta_{2} & (++-) \\
\Delta_{3}=3-\Delta_{1}-\Delta_{2} & (+++)
\end{array}
$$

When either of these conditions is satisfied by the seed correlator, the action of $W_{12}^{++}$does not reproduce the correct result. However, $W_{12}^{--}$works as it can be checked that it does not change the type of divergence. 


\section{Allowed parity-odd 3-point functions in various dimensions}

Parity-odd structures for three-point CFT correlators can exist in simple theories such as the free fermion theory in three-dimensions and in CFTs which do not have a parity symmetry. Such structures change sign under inversion and they always involve the antisymmetric epsilon tensor. The existence, or non-existence, of such correlators in various dimensions is easily seen in the embedding space formalism [66, 67]. In embedding space, $d$-dimensional parity-odd correlators are characterised by $(d+2)$-dimensional epsilon tensor. Although we restrict our attention to correlators involving spin-zero, spin-one and spin-two currents, the analysis below can be easily generalised to parity-odd correlators involving traceless symmetric operators in various dimensions. We will not consider the case of 3-point correlators of mixed symmetry primary operators which exist in $d \geq 4$ and for which parity-odd structures are possible. We refer the reader to appendix A for some details on the embedding space formalism.

One of the constraints on correlators with a spinning operator is that they are transverse, i.e. the epsilon structure should be invariant under $Z_{i} \rightarrow Z_{i}+\beta X_{i}$. This constrains the possible epsilon structures one can have in a given dimension.

\section{$3.1 \quad$ Three-dimensions}

In three-dimensions, transversality implies that the parity-odd invariants that can exist are:

$$
\epsilon\left(Z_{1} Z_{2} X_{1} X_{2} X_{3}\right), \quad \epsilon\left(Z_{2} Z_{3} X_{1} X_{2} X_{3}\right), \quad \epsilon\left(Z_{3} Z_{1} X_{1} X_{2} X_{3}\right)
$$

This immediately implies that the following parity-odd correlation function is zero:

$$
\left\langle J_{s} O_{\Delta_{1}} O_{\Delta_{2}}\right\rangle_{\text {odd }}=0
$$

where $O_{\Delta_{1}}, O_{\Delta_{2}}$ are scalar operators with dimensions as indicated and $J_{s}$ is a spin $s$ operator. However, correlators of the form $\left\langle J_{s_{1}} J_{s_{2}} O_{\Delta}\right\rangle_{\text {odd }}$ and $\left\langle J_{s_{1}} J_{s_{2}} J_{s_{3}}\right\rangle_{\text {odd }}$ are non-zero. See [65] for details. In section 4.1, we will explicitly show that, in momentum space:

$$
\left\langle J_{\mu} O O\right\rangle_{\text {odd }}=0 .
$$

We will also calculate other parity-odd three-point functions in subsequent sections.

\section{$3.2 \quad$ Four-dimensions}

In four-dimensions, transversality allows only the following parity-odd invariant:

$$
\epsilon\left(Z_{1} Z_{2} Z_{3} X_{1} X_{2} X_{3}\right)
$$

This implies that in four-dimensions the following correlators are zero:

$$
\left\langle J_{s_{1}} O O\right\rangle_{\text {odd }}=0, \quad\left\langle J_{s_{1}} J_{s_{2}} O\right\rangle_{\text {odd }}=0
$$

Correlators are further constrained by symmetry requirements. For example,

$$
\langle J J T\rangle_{\text {odd }}=0, \quad\langle T T T\rangle_{\text {odd }}=0 .
$$

See appendix F for details. While these correlators are zero, $\left\langle J_{\mu}^{a} J_{\nu}^{b} J_{\rho}^{c}\right\rangle$ can be non-zero. 


\subsection{Five-dimensions and above}

In five-dimensions, the only parity-odd invariant allowed by transversality is:

$$
\epsilon\left(Z_{1} Z_{2} Z_{3} X_{1} X_{2} X_{3} X_{4}\right)
$$

The structure of the contracted epsilon tensor makes it clear that we cannot have any parity-odd three-point function of symmetric traceless operators in five dimensions.

\section{Momentum space parity-odd 3-point functions in three-dimensions us- ing conformal Ward identity}

In this section, we will use the direct approach of solving the PDEs for the form factors which result from the momentum space conformal Ward identities. We will illustrate this using the correlators $\langle J O O\rangle$ and $\langle J J O\rangle$ as examples. This is an extension of the analysis in [2] to the parity-odd sector. We also discuss the divergences that can arise, and the counter-terms that regulate them. We conclude this section with a small discussion on the difficulties in using this method to compute correlators involving more general spinning operators.

\section{$4.1\left\langle J^{\mu} O O\right\rangle_{\text {odd }}$}

Let us consider the parity-odd part of the $\left\langle J^{\mu} O O\right\rangle$ correlator where $J^{\mu}$ is a conserved current and $O$ has scaling dimension 2. A suitable ansatz for the correlator is:

$$
\left\langle O^{a}\left(k_{1}\right) O^{b}\left(k_{2}\right) J^{\mu c}\left(k_{3}\right)\right\rangle_{\text {odd }}=A\left(k_{1}, k_{2}, k_{3}\right) f^{a b c} \epsilon^{\mu k_{1} k_{2}}
$$

Throughout this paper, we use notations such as $\epsilon^{\mu \nu k_{1}}$ and $\epsilon^{\mu k_{1} k_{2}}$ and they stand for the epsilon tensor contracted with the momenta:

$$
\epsilon^{\mu \nu k_{1}}=\epsilon^{\mu \nu \rho} k_{1 \rho}, \quad \epsilon^{\mu k_{1} k_{2}}=\epsilon^{\mu \nu \rho} k_{1 \nu} k_{2 \rho}
$$

We have considered $J^{\mu}$ to be in the third position as this makes the action of $K^{\kappa}$ simpler (2.4). Without non-abelian indices the correlator is zero as $\left(k_{1} \leftrightarrow k_{2}\right)$ exchange symmetry would require $A\left(k_{1}, k_{2}\right)=-A\left(k_{2}, k_{1}\right)$. After acting with $K^{\kappa}$, the primary Ward identities are given by:

$$
\begin{aligned}
& \frac{\partial^{2} A}{\partial k_{1}^{2}}-\frac{\partial^{2} A}{\partial k_{3}^{2}}-\frac{2}{k_{3}} \frac{\partial A}{\partial k_{3}}=0 \\
& \frac{\partial^{2} A}{\partial k_{2}^{2}}-\frac{\partial^{2} A}{\partial k_{3}^{2}}-\frac{2}{k_{3}} \frac{\partial A}{\partial k_{3}}=0
\end{aligned}
$$

The above differential equations can be solved in terms of triple- $K$ integrals (2.5) to get:

$$
A\left(k_{1}, k_{2}, k_{3}\right)=c_{1} I_{\frac{3}{2}\left\{\frac{1}{2} \frac{1}{2}-\frac{1}{2}\right\}}=c_{1} \frac{1}{k_{3}\left(k_{1}+k_{2}+k_{3}\right)}
$$

The correlator also satisfies an independent secondary Ward identity given by:

$$
2 A+\frac{k_{1}^{2}-k_{2}^{2}+k_{3}^{2}}{k_{3}} \frac{\partial A}{\partial k_{3}}+2 k_{1} \frac{\partial A}{\partial k_{1}}=0
$$


The right hand side of the above equation is proportional to the 2-point function $\left\langle\mathrm{O}_{2}(k) \mathrm{O}_{2}(-k)\right\rangle$. However, the scalar two-point function has no parity-odd contribution and thus the R.H.S. of (4.5) is zero. Substituting (4.4) into (4.5) gives

$$
c_{1}=0 \Longrightarrow A\left(k_{1}, k_{2}, k_{3}\right)=0
$$

Thus we conclude that:

$$
\left\langle O^{a}\left(k_{1}\right) O^{b}\left(k_{2}\right) J^{\mu c}\left(k_{3}\right)\right\rangle_{\text {odd }}=0
$$

This result can be generalised to scalar operators of arbitrary scaling dimensions.

\section{$4.2\left\langle J^{\mu} J^{\nu} O\right\rangle_{\text {odd }}$}

Here we will consider the parity-odd part of the correlator $\left\langle J^{\mu} J^{\nu} O\right\rangle$. We start with the following ansatz for the correlator:

$$
\left\langle J^{\mu}\left(k_{1}\right) J^{\nu}\left(k_{2}\right) O\left(k_{3}\right)\right\rangle_{\text {odd }}=\pi_{\alpha}^{\mu}\left(k_{1}\right) \pi_{\beta}^{\nu}\left(k_{2}\right)\left[\widetilde{A}\left(k_{1}, k_{2}, k_{3}\right) \epsilon^{\alpha k_{1} k_{2}} k_{1}^{\beta}+\widetilde{B}\left(k_{1}, k_{2}, k_{3}\right) \epsilon^{\beta k_{1} k_{2}} k_{2}^{\alpha}\right]
$$

where the orthogonal projector $\pi_{\nu}^{\mu}(p)$ is given by:

$$
\pi_{\mu}^{\nu}(p) \equiv \delta_{\mu}^{\nu}-\frac{p^{\nu} p_{\mu}}{p^{2}}
$$

The ansatz (4.8) is chosen such that the correlator is transverse with respect to $k_{1}^{\mu}$ and $k_{2}^{\nu}$. Demanding symmetry under the exchange: $\left(k_{1}, \mu\right) \leftrightarrow\left(k_{2}, \nu\right)$ gives the following relation between the form factors:

$$
\widetilde{A}\left(k_{1}, k_{2}, k_{3}\right)=-\widetilde{B}\left(k_{2}, k_{1}, k_{3}\right)
$$

Using the definition of projectors (4.9), the ansatz (4.8) expands to the following:

$$
\begin{aligned}
\left\langle J^{\mu}\left(k_{1}\right) J^{\nu}\left(k_{2}\right) O\left(k_{3}\right)\right\rangle_{\text {odd }}= & \widetilde{A}\left(k_{1}, k_{2}, k_{3}\right) \epsilon^{\mu k_{1} k_{3}}\left[\frac{\left(k_{1}^{\nu}+k_{3}^{\nu}\right)\left(k_{1}^{2}+k_{1} \cdot k_{3}\right)}{k_{2}^{2}}-k_{1}^{\nu}\right] \\
& +\widetilde{B}\left(k_{1}, k_{2}, k_{3}\right) \epsilon^{\nu k_{1} k_{3}}\left[\left(k_{1}^{\mu}+k_{3}^{\mu}\right)-\frac{k_{1}^{\mu}\left(k_{1}^{2}+k_{1} \cdot k_{3}\right)}{k_{1}^{2}}\right]
\end{aligned}
$$

where we have used momentum conservation to choose $k_{1}$ and $k_{3}$ as the independent momenta.

We now use Schouten identities (C.3) and (C.4) to get rid of the $\epsilon^{\mu k_{1} k_{2}}$ tensor structure and re-express the ansatz in (4.11) as:

$$
\left\langle J^{\mu}\left(k_{1}\right) J^{\nu}\left(k_{2}\right) O\left(k_{3}\right)\right\rangle_{\text {odd }}=-\epsilon^{\nu k_{1} k_{3}}\left(A k_{1}^{\mu}-B k_{1}^{\mu}-B k_{3}^{\mu}\right)-\left(\epsilon^{\mu \nu k_{1}}+\epsilon^{\mu \nu k_{3}}\right)\left(A k_{1}^{2}+B\left(k_{1} \cdot k_{2}\right)\right)
$$

where the new form factors $A\left(k_{1}, k_{2}, k_{3}\right)$ and $B\left(k_{1}, k_{2}, k_{3}\right)$ are given in terms of $\widetilde{A}\left(k_{1}, k_{2}, k_{3}\right)$ and $\widetilde{B}\left(k_{1}, k_{2}, k_{3}\right)$ as follows:

$$
\begin{aligned}
& A\left(k_{1}, k_{2}, k_{3}\right)=\widetilde{A}\left(k_{1}, k_{2}, k_{3}\right)+\widetilde{B}\left(k_{1}, k_{2}, k_{3}\right)+\widetilde{B}\left(k_{1}, k_{2}, k_{3}\right) \frac{k_{1} \cdot k_{3}}{k_{1}^{2}} \\
& B\left(k_{1}, k_{2}, k_{3}\right)=\widetilde{B}\left(k_{1}, k_{2}, k_{3}\right)-\widetilde{A}\left(k_{1}, k_{2}, k_{3}\right) \frac{k_{1} \cdot k_{2}}{k_{2}^{2}}
\end{aligned}
$$


Note that the exchange symmetry (4.10) continues to hold between $A$ and $B$ :

$$
A\left(k_{1}, k_{2}, k_{3}\right)=-B\left(k_{2}, k_{1}, k_{3}\right)
$$

We will now obtain the primary and secondary Ward identities that $A\left(k_{1}, k_{2}, k_{3}\right)$ and $B\left(k_{1}, k_{2}, k_{3}\right)$ satisfy, by letting the generator of special conformal transformations $K^{\kappa}(2.4)$ act on the ansatz (4.12):

$$
\begin{aligned}
K^{\kappa}\left\langle J^{\mu}\left(k_{1}\right) J^{\nu}\left(k_{3}\right) O\left(k_{2}\right)\right\rangle_{\text {odd }}= & -2 \frac{\partial}{\partial k_{1}^{\kappa}}-2 k_{1}^{\alpha} \frac{\partial}{\partial k_{1}^{\alpha}} \frac{\partial}{\partial k_{1}^{\kappa}}+k_{1, \kappa} \frac{\partial}{\partial k_{1}^{\alpha}} \frac{\partial}{\partial k_{1 \alpha}} \\
& \left.+2\left(\Delta_{3}-3\right) \frac{\partial}{\partial k_{3}^{\kappa}}-2 k_{3}^{\alpha} \frac{\partial}{\partial k_{3}^{\alpha}} \frac{\partial}{\partial k_{3}^{\kappa}}+k_{3, \kappa} \frac{\partial}{\partial k_{3}^{\alpha}} \frac{\partial}{\partial k_{3 \alpha}}\right] \\
\times & \left\langle J^{\mu}\left(k_{1}\right) J^{\nu}\left(k_{2}\right) O\left(k_{3}\right)\right\rangle \\
+ & 2\left(\delta^{\mu \kappa} \frac{\partial}{\partial k_{1}^{\alpha}}-\delta_{\alpha}^{\kappa} \frac{\partial}{\partial k_{1, \mu}}\right)\left\langle J^{\alpha}\left(k_{1}\right) J^{\nu}\left(k_{2}\right) O\left(k_{3}\right)\right\rangle
\end{aligned}
$$

Note that by choosing $k_{1}$ and $k_{3}$ as the independent momenta, we got rid of one set of terms in the generator $K^{\kappa}$ that mixes with the index structure of the correlator.

The primary Ward identities satisfied by $A\left(k_{1}, k_{2}, k_{3}\right)$ are given by:

$$
\begin{array}{r}
\frac{\partial^{2} A}{\partial k_{1}^{2}}+\frac{\partial^{2} A}{\partial k_{3}^{2}}+\frac{2 k_{1}}{k_{3}} \frac{\partial^{2} A}{\partial k_{1} \partial k_{3}}+\frac{2 k_{2}}{k_{3}} \frac{\partial^{2} A}{\partial k_{2} \partial k_{3}}+\frac{2}{k_{1}} \frac{\partial A}{\partial k_{1}}+\frac{8}{k_{3}} \frac{\partial A}{\partial k_{3}}=0 \\
\frac{\partial^{2} A}{\partial k_{3}^{2}}+\frac{\partial^{2} A}{\partial k_{2}^{2}}+\frac{2 k_{1}}{k_{3}} \frac{\partial^{2} A}{\partial k_{1} \partial k_{3}}+\frac{2 k_{2}}{k_{3}} \frac{\partial^{2} A}{\partial k_{2} \partial k_{3}}+\frac{8}{k_{3}} \frac{\partial A}{\partial k_{3}}=0
\end{array}
$$

Similarly, the equations for $B\left(k_{1}, k_{2}, k_{3}\right)$ are given by:

$$
\begin{array}{r}
\frac{\partial^{2} B}{\partial k_{2}^{2}}+\frac{\partial^{2} B}{\partial k_{3}^{2}}+\frac{2 k_{1}}{k_{3}} \frac{\partial^{2} B}{\partial k_{1} \partial k_{3}}+\frac{2 k_{2}}{k_{3}} \frac{\partial^{2} B}{\partial k_{2} \partial k_{3}}+\frac{2}{k_{2}} \frac{\partial B}{\partial k_{2}}+\frac{8}{k_{3}} \frac{\partial B}{\partial k_{3}}=0 \\
\frac{\partial^{2} B}{\partial k_{3}^{2}}+\frac{\partial^{2} B}{\partial k_{1}^{2}}+\frac{2 k_{1}}{k_{3}} \frac{\partial^{2} B}{\partial k_{1} \partial k_{3}}+\frac{2 k_{2}}{k_{3}} \frac{\partial^{2} B}{\partial k_{2} \partial k_{3}}+\frac{8}{k_{3}} \frac{\partial B}{\partial k_{3}}=0
\end{array}
$$

The general solution to both the primary Ward identities can be found in terms of triple- $K$ integrals (2.5). We solve for $\beta_{1}, \beta_{2}, \beta_{3}$ by substituting the triple- $K$ integral into the primary Ward identities, and obtain:

$$
\begin{aligned}
& A \propto I_{\alpha\left\{-\frac{1}{2}, \frac{1}{2}, \Delta_{3}-\frac{3}{2}\right\}} \\
& B \propto I_{\alpha\left\{\frac{1}{2},-\frac{1}{2}, \Delta_{3}-\frac{3}{2}\right\}}
\end{aligned}
$$

The unknown $\alpha$ is determined using the dilatation Ward identity. The action of the dilatation Ward identity on the ansatz gives the degree of the form factors:

$$
\begin{aligned}
& \operatorname{deg}(A)=1+\Delta_{3}-N_{A} \\
& \operatorname{deg}(B)=1+\Delta_{3}-N_{B}
\end{aligned}
$$

where $N_{A}$ and $N_{B}$ are the tensorial dimensions of $A$ and $B$, defined as the number of momenta that multiply the form factor in the ansatz. We see from (4.12) and (4.8) that 
$N_{A}=N_{B}=3$. Similarly, we impose the dilatation Ward identity on the triple- $K$ integral and get:

$$
\operatorname{deg}\left(I_{\alpha\left\{\beta_{j}\right\}}\right)=\beta_{1}+\beta_{2}+\beta_{3}-\alpha-1
$$

This must equal the degree of the form factors $A$ and $B$ (4.19) giving us:

$$
\alpha=1-\Delta_{3}+\sum_{i=1}^{3} \beta_{i}
$$

Thus we obtain:

$$
\begin{aligned}
& A=c_{1} I_{-\frac{1}{2}\left\{-\frac{1}{2}, \frac{1}{2}, \Delta_{3}-\frac{3}{2}\right\}} \\
& B=c_{2} I_{-\frac{1}{2}\left\{\frac{1}{2},-\frac{1}{2}, \Delta_{3}-\frac{3}{2}\right\}}
\end{aligned}
$$

where $c_{1}$ and $c_{2}$ are undetermined constants.

We now present the explicit expressions for the two form factors for a few values of the scaling dimension of the scalar operator $O$. When the scalar operator has $\Delta_{3}=1$, we have,

$$
\begin{aligned}
& A\left(k_{1}, k_{2}, k_{3}\right)=c_{1} \sqrt{\frac{\pi^{3}}{8}} \frac{1}{k_{1} k_{3}\left(k_{1}+k_{2}+k_{3}\right)^{2}}, \\
& B\left(k_{1}, k_{2}, k_{3}\right)=c_{2} \sqrt{\frac{\pi^{3}}{8}} \frac{1}{k_{2} k_{3}\left(k_{1}+k_{2}+k_{3}\right)^{2}}
\end{aligned}
$$

For $\Delta_{3}=2$ :

$$
\begin{aligned}
& A\left(k_{1}, k_{2}, k_{3}\right)=c_{1} \sqrt{\frac{\pi^{3}}{8}} \frac{1}{k_{1}\left(k_{1}+k_{2}+k_{3}\right)^{2}}, \\
& B\left(k_{1}, k_{2}, k_{3}\right)=c_{2} \sqrt{\frac{\pi^{3}}{8}} \frac{1}{k_{2}\left(k_{1}+k_{2}+k_{3}\right)^{2}} .
\end{aligned}
$$

When $\Delta_{3}=3$ :

$$
\begin{aligned}
& A\left(k_{1}, k_{2}, k_{3}\right)=c_{1} \sqrt{\frac{\pi^{3}}{8}} \frac{k_{1}+k_{2}+2 k_{3}}{k_{1}\left(k_{1}+k_{2}+k_{3}\right)^{2}} \\
& B\left(k_{1}, k_{2}, k_{3}\right)=c_{2} \sqrt{\frac{\pi^{3}}{8}} \frac{k_{1}+k_{2}+2 k_{3}}{k_{2}\left(k_{1}+k_{2}+k_{3}\right)^{2}} .
\end{aligned}
$$

We will now look at the secondary Ward identities to fix the undetermined constants $c_{1}$ and $c_{2}$ in (4.22).

There is one independent secondary Ward identity in this case which leaves just one independent, undetermined constant. The identity is given by:

$$
\frac{k_{1}^{2}}{k_{2}} \frac{\partial A}{\partial k_{2}}+k_{1} \frac{\partial B}{\partial k_{1}}=0
$$

Substituting the solutions for the form factors from (4.22) in this equation we get:

$$
c_{2}=-c_{1}
$$

which is exactly what is expected from symmetry considerations. 


\subsubsection{Divergences and renormalization}

We saw in equations (4.23), (4.24) and (4.25) that the triple- $K$ integral is convergent for $\Delta=1,2,3$. For $\Delta_{3}>3$, the integral is singular in the regulator and in some cases, we will require counter-terms to remove this divergence.

The generating functional for the theory is defined as:

$$
Z=\int D \phi \exp \left(-\int d^{3} x\left(S_{\phi}\left[A_{\mu}, g^{\mu \nu}\right]+\sqrt{g} O \phi+J^{\mu} A_{\mu}\right)\right)
$$

where $\phi$ and $A_{\mu}$ are sources of the scalar operator and the conserved spin-one current respectively. For certain classes of divergences, the generating functional is modified by counter-terms. We classify the values of $\Delta_{3}$ into two classes based on the kinds of divergences that occur.

$\boldsymbol{\Delta}_{\mathbf{3}}=\mathbf{4}+\mathbf{2 n}$ where $\boldsymbol{n} \in \mathbb{Z}_{\geq \mathbf{0}}$. When $\Delta_{3}=4$, i.e. $n=0,(2.8)$ is satisfied for the choice of signs given by $(+--)$. When $n>0$, it is satisfied for the choice of signs $(+--)$ and $(-+-)$. We choose to work in a convenient regularisation scheme where we shift $\Delta_{3}$ as $\Delta_{3} \rightarrow \Delta_{3}+\epsilon$ and keep the dimension $d$ of the space-time and the conformal dimensions $\Delta_{1}$ and $\Delta_{2}$ as in the unregulated theory.

To remove this singularity, we look at the following parity-odd counter-term from (2.10)

$$
S_{c t}=a(\epsilon) \int d^{3} x \mu^{\epsilon} \epsilon^{\mu \nu \lambda} F_{\mu \nu} J_{\lambda} \square^{n} \phi
$$

where $\mu$ is the renormalization scale. After taking suitable functional derivatives, the contribution to the correlator from this counter-term is given by

$$
\begin{aligned}
\left\langle J^{\mu}\left(x_{1}\right) J^{\nu}\left(x_{2}\right) O\left(x_{3}\right)\right\rangle_{c t}=-a(\epsilon)[ & \square^{n}\left(\delta^{3}\left(x_{2}-x_{3}\right) \epsilon^{\rho \nu \lambda} \partial_{2 \rho}\left\langle J^{\lambda}\left(x_{1}\right) J^{\mu}\left(x_{3}\right)\right\rangle\right) \\
& \left.-\square^{n}\left(\delta^{3}\left(x_{1}-x_{3}\right) \epsilon^{\rho \mu \lambda} \partial_{1 \rho}\left\langle J^{\lambda}\left(x_{3}\right) J^{\nu}\left(x_{2}\right)\right\rangle\right)\right]
\end{aligned}
$$

A Fourier transform of the above gives:

$$
\begin{aligned}
\left\langle J^{\mu}\left(k_{1}\right) J^{\nu}\left(k_{2}\right) O\left(k_{3}\right)\right\rangle_{c t} & =-a(\epsilon)\left(k_{2}^{2 n} \epsilon^{\nu k_{2} \lambda} \pi_{\lambda}^{\mu}\left(k_{1}\right) k_{1}-k_{1}^{2 n} \epsilon^{\mu k_{1} \lambda} \pi_{\lambda}^{\nu}\left(k_{2}\right) k_{2}\right) \\
& =-a(\epsilon)\left[k_{2}^{2 n} k_{1}\left(\epsilon^{\mu \nu k_{2}}+\frac{\epsilon^{\nu k_{1} k_{2}} k_{1}^{\mu}}{k_{1}^{2}}\right)-k_{1}^{2 n} k_{2}\left(\epsilon^{\mu \nu k_{1}}+\frac{\epsilon^{\mu k_{1} k_{2}} k_{2}^{\nu}}{k_{2}^{2}}\right)\right] \mu^{-\epsilon}
\end{aligned}
$$

where we used the following 2-point function: ${ }^{6}$

$$
\left\langle J^{\mu}(k) J_{\nu}(-k)\right\rangle=\pi_{\nu}^{\mu}(k) k
$$

Using Schouten identities (C.3) and (C.4), the ansatz for the correlator can be written as

$$
\left\langle J^{\mu}\left(k_{1}\right) J^{\nu}\left(k_{2}\right) O\left(k_{3}\right)\right\rangle=A_{1}\left(\epsilon^{\mu \nu k_{2}} k_{1}^{2}+\epsilon^{\nu k_{1} k_{2}} k_{1}^{\mu}\right)+A_{2}\left(\epsilon^{\mu \nu k_{1}} k_{2}^{2}+\epsilon^{\mu k_{1} k_{2}} k_{2}^{\nu}\right)
$$

\footnotetext{
${ }^{6}$ The counter-term that we used (4.29) could also contribute to the parity-even part of $\langle J J O\rangle$ since the $\langle J J\rangle$ 2-point-function has a parity-odd contribution.
} 
When $\Delta_{3}=4$ the singular part of the regularised form factors are given by

$$
A_{1}\left(k_{1}, k_{2}, k_{3}\right)=\frac{1}{k_{1} \epsilon}, \quad A_{2}\left(k_{1}, k_{2}, k_{3}\right)=-\frac{1}{k_{2} \epsilon}
$$

The contribution of the counter-term (4.31) to the correlator in this case $\left(\Delta_{3}=4\right.$, or equivalently $n=0$ ) is given by:

$$
\left\langle J^{\mu}\left(k_{1}\right) J^{\nu}\left(k_{2}\right) O\left(k_{3}\right)\right\rangle_{c t}=-a(\epsilon)\left[k_{1}\left(\epsilon^{\mu \nu k_{2}}+\frac{\epsilon^{\nu k_{1} k_{2}} k_{1}^{\mu}}{k_{1}^{2}}\right)-k_{2}\left(\epsilon^{\mu \nu k_{1}}+\frac{\epsilon^{\mu k_{1} k_{2}} k_{2}^{\nu}}{k_{2}^{2}}\right)\right] \mu^{-\epsilon}
$$

Comparing (4.35) and (4.33) along with (4.34) we see that choosing $a(\epsilon)=1 / \epsilon$ cancels the singular part of the correlator. After removing the divergences, the resulting form factor is given by:

$$
A_{1}\left(k_{1}, k_{2}, k_{3}\right)=c_{1} \frac{3}{k_{1}} \log \left(\frac{k_{1}+k_{2}+k_{3}}{\mu}\right)-c_{1} \frac{k_{3}^{2}+3 k_{3}\left(k_{1}+k_{2}+k_{3}\right)}{k_{1}\left(k_{1}+k_{2}+k_{3}\right)^{2}}
$$

The second form factor is obtained by the following exchange:

$$
A_{2}\left(k_{1}, k_{2}, k_{3}\right)=-A_{1}\left(k_{2}, k_{1}, k_{3}\right)
$$

The anomalous dilatation Ward identity takes the form:

$$
\mu \frac{\partial A_{1}}{\partial \mu}=-\frac{c_{1}}{k_{1}}
$$

$\boldsymbol{\Delta}_{\mathbf{3}}=\mathbf{5}+\mathbf{2 n}$ where $\boldsymbol{n} \in \mathbb{Z}_{\geq \mathbf{0}}$. In this case, (2.8) is satisfied for the choice of signs given by $(---)$ and $(++-)$. Although we have both an ultra-local and a non-local divergence here, the term at $\mathcal{O}(1 / \epsilon)$ is non-local in the momenta and therefore the divergence can be cancelled by multiplying with a constant of $\mathcal{O}(\epsilon)$ and then taking the limit $\epsilon \rightarrow 0$. In particular, when $\Delta_{3}=5$, the divergent term can be calculated to be:

$$
A_{1}\left(k_{1}, k_{2}, k_{3}\right)=c_{1}(\epsilon) \frac{k_{1}+k_{2}}{k_{1} \epsilon}+O\left(\epsilon^{0}\right)
$$

Choosing $c_{1}$ to be $O(\epsilon)$, the resulting form factor is:

$$
A_{1}\left(k_{1}, k_{2}, k_{3}\right)=c_{1}^{(1)} \frac{k_{1}+k_{2}}{k_{1}}
$$

where $c_{1}^{(1)}$ is $\mathcal{O}(0)$ in $\epsilon$.

It can be easily checked that this form factor satisfies non-anomalous Ward identities and that scale invariance is not broken.

\subsection{Non-Abelian $\langle J J O\rangle_{\text {odd }}$}

If we add a colour index to all the three operators in the correlator such that an exchange of two colour indices gives rise to a minus sign, then the symmetry between the form factors in (4.8) changes to:

$$
\widetilde{A}\left(k_{1}, k_{2}, k_{3}\right)=\widetilde{B}\left(k_{2}, k_{1}, k_{3}\right)
$$


The solution for $\left\langle J^{\mu a}\left(k_{1}\right) J^{\nu b}\left(k_{2}\right) O_{\Delta}^{c}\left(k_{3}\right)\right\rangle$, when $\Delta=3$ is then given by:

$$
\begin{aligned}
\left\langle J^{\mu a}\left(k_{1}\right) J^{\nu b}\left(k_{2}\right) O_{\Delta}^{c}\left(k_{3}\right)\right\rangle_{\text {odd }}= & f^{a b c}\left[\epsilon^{\nu k_{1} k_{2}}\left(\frac{k_{1}+k_{2}+2 k_{3}}{k_{1}\left(k_{1}+k_{2}+k_{3}\right)^{2}} k_{1}^{\mu}+\frac{k_{1}+k_{2}+2 k_{3}}{k_{2}\left(k_{1}+k_{2}+k_{3}\right)^{2}} k_{2}^{\mu}\right)\right. \\
& \left.+\epsilon^{\mu \nu k_{2}}\left(\frac{k_{1}+k_{2}+2 k_{3}}{k_{1}\left(k_{1}+k_{2}+k_{3}\right)^{2}} k_{1}^{2}+\frac{k_{1}+k_{2}+2 k_{3}}{k_{2}\left(k_{1}+k_{2}+k_{3}\right)^{2}}\left(k_{1} \cdot k_{2}\right)\right)\right]
\end{aligned}
$$

It can be checked that the above solution is symmetric under $(1 \leftrightarrow 2)$ exchange upon using suitable Schouten identities.

In principle one can compute parity-odd correlation functions of higher spin operators using the approach described in this section following [2]. However, it soon gets difficult to find out the independent tensor structures after the application of the generator of special conformal transformations, due to non-trivial Schouten identities that relate various tensor structures. We will now resort to the technique of using weight-shifting and spin-raising operators to compute parity-odd correlation functions.

\section{Parity-odd spin-raising \& weight-shifting operators in three- dimensions}

In this section we construct parity-odd spin-raising and weight-shifting operators in momentum space. We then illustrate how these operators can be used to calculate the parity-odd part of the $\langle J J O\rangle$ correlator.

\subsection{Parity-odd operators}

We consider a parity-odd operator which raises the spins of the operators at points 1 and 2 and lowers the weight of the operator at point 2. In embedding space, this operator is defined $^{7}$ as

$$
\widetilde{D}_{12} \equiv \epsilon\left(Z_{1}, Z_{2}, X_{1}, X_{2}, \frac{\partial}{\partial X_{1}}\right)
$$

based on requirements of transversality and interiority [67]. In position space, the operator takes the form:

$$
\widetilde{D}_{12}=\frac{1}{2}\left(\epsilon^{i j k-+} z_{1 i} z_{2 j} x_{12 k} D_{1}-\epsilon^{i j k-+}\left[\frac{\left(x_{1}^{2}-x_{2}^{2}\right)}{2} z_{1 j} z_{2 k}+x_{12 j} z_{1 k}\left(z_{2} \cdot x_{2}\right)+z_{2 j} x_{12 k}\left(z_{1} \cdot x_{1}\right)\right] P_{1 i}\right)
$$

where $\epsilon^{i j k-+} \equiv \epsilon^{i j k}$. Performing a Fourier transform we get in momentum space:

$$
\begin{aligned}
\widetilde{D}_{12}=-\frac{1}{2}[ & \epsilon\left(z_{1} z_{2} K_{12}^{-}\right)\left(\Delta_{1}-d-k_{1} \cdot \frac{\partial}{\partial k_{1}}\right) \\
& \left.\quad+\frac{K_{12}^{-} K_{12}^{+}}{2} \epsilon\left(k_{1} z_{1} z_{2}\right)+\epsilon\left(k_{1} K_{12}^{-} z_{1}\right)\left(z_{2} \cdot \frac{\partial}{\partial k_{2}}\right)+\epsilon\left(k_{1} z_{2} K_{12}^{-}\right)\left(z_{1} \cdot \frac{\partial}{\partial k_{1}}\right)\right]
\end{aligned}
$$

where $K_{12}^{+}$and $K_{12}^{-}$are defined in appendix E.

\footnotetext{
${ }^{7}$ We would like to thank the referee for pointing out that this form for the weight-shifting operator is correct only when acting on scalar correlators. Otherwise, there would be an additional term containing $\partial / \partial Z$ in the correct general form. However, in all of our constructions in this paper we will only need this operator to act on scalar correlators, so no problem arises.
} 
The above operator acts on a momentum space correlator with a momentum conserving delta function. In its present form, it will be tedious to take the above operator past the delta function. Consider now the following commutator:

$$
\left[k_{1}^{\mu}+k_{2}^{\mu}+k_{3}^{\mu}, \widetilde{D}_{12}\right]=-\frac{1}{2}\left[\epsilon\left(z_{1} z_{2} K_{12}^{-}\right) k_{1}^{\mu}-K_{12}^{-\mu} \epsilon\left(k_{1} z_{1} z_{2}\right)-\epsilon\left(k_{1} K_{12}^{-} z_{1}\right) z_{2}^{\mu}-\epsilon\left(k_{1} z_{2} K_{12}^{-}\right) z_{1}^{\mu}\right]
$$

The above commutator vanishes on a three-point function due to momentum conservation. Thus we have the following action on 3-point functions:

$$
\epsilon\left(z_{1} z_{2} K_{12}^{-}\right) k_{1}^{\mu}-K_{12}^{-\mu} \epsilon\left(k_{1} z_{1} z_{2}\right)-\epsilon\left(k_{1} K_{12}^{-} z_{1}\right) z_{2}^{\mu}-\epsilon\left(k_{1} z_{2} K_{12}^{-}\right) z_{1}^{\mu}=0
$$

We contract the above equation with $\frac{\partial}{\partial k_{1}^{\mu}}$ from the right to get the Schouten identity:

$$
\begin{aligned}
& \epsilon\left(z_{1} z_{2} K_{12}^{-}\right) k_{1} \cdot \frac{\partial}{\partial k_{1}}+\epsilon\left(z_{1} z_{2} K_{12}^{-}\right)-\left(K_{12}^{-} \cdot \frac{\partial}{\partial k_{1}}\right) \epsilon\left(k_{1} z_{1} z_{2}\right)-\epsilon\left(k_{1} K_{12}^{-} z_{1}\right)\left(z_{2} \cdot \frac{\partial}{\partial k_{1}}\right) \\
&-\epsilon\left(k_{1} z_{2} K_{12}^{-}\right)\left(z_{1} \cdot \frac{\partial}{\partial k_{1}}\right)=0
\end{aligned}
$$

We use this to rewrite (5.3) as:

$$
\widetilde{D}_{12}=\epsilon^{z_{1} z_{2} k_{1}} W_{12}^{--}+\epsilon^{z_{1} k_{1} K_{12}^{-}}\left(\vec{z}_{2} \cdot \vec{K}_{12}^{-}\right)+\left(2-\Delta_{1}\right) \epsilon^{z_{1} z_{2} K_{12}^{-}}
$$

where $W_{12}^{--}$is defined in appendix E. Note that the operator defined in (5.7) is explicitly translation invariant. ${ }^{8}$

We will use $\widetilde{D}_{12}$ in (5.7) to compute $\left\langle J J O_{\Delta}\right\rangle_{\text {odd }},\langle J J J\rangle_{\text {odd }}$ and $\left\langle T T O_{\Delta}\right\rangle_{\text {odd }}{ }^{9}$ starting from a scalar-seed. We can also construct $\widetilde{D}_{23}$ and $\widetilde{D}_{31}$ to get operators that act on points 2 and 3 and points 3 and 1 , respectively. We will require them in the computation of $\langle J J J\rangle_{\text {odd }}$ as the correlator has cyclic symmetry.

We will now construct other parity-odd weight-shifting and spin-raising operators that are useful. Let us consider the following:

$$
\begin{aligned}
\widetilde{D}_{1} \equiv & \epsilon\left(Z_{1}, X_{1}, \frac{\partial}{\partial X^{1}}, X_{2}, \frac{\partial}{\partial X_{2}}\right)+\epsilon\left(Z_{1}, X_{1}, \frac{\partial}{\partial X^{1}}, Z_{2}, \frac{\partial}{\partial Z_{2}}\right) \\
= & \frac{1}{2}\left\{\epsilon^{i j k}\left[z_{1 i} x_{12 k}\left(D_{2} P_{1 j}-D_{1} P_{2 j}\right)+\left(x_{1} \cdot z_{1}\right) x_{12 k} P_{1 i} P_{2 j}-\frac{\left(x_{1}^{2}-x_{2}^{2}\right)}{2} z_{1 k} P_{1 i} P_{2 j}\right]\right\} \\
& +\frac{1}{2}\left\{\epsilon^{i j k}\left[\left(x_{1} \cdot z_{1}\right) z_{2 j} \frac{\partial}{\partial z_{2}^{k}}+\left(x_{2} \cdot z_{2}\right) z_{1 k} \frac{\partial}{\partial z_{2}^{j}}+z_{1 j} z_{2 k}\left(x_{2} \cdot \frac{\partial}{\partial z_{2}}\right)\right] P_{1 i}\right\} \\
& -\frac{1}{2} \epsilon^{i j k}\left[z_{1 i} z_{2 j} \frac{\partial}{\partial z^{2 k}}\right] D_{1} .
\end{aligned}
$$

\footnotetext{
${ }^{8}$ Translational invariance of an operator implies in momentum space that its operation on the momentum conserving delta-function is zero. This happens when the operator is only a function of $K_{i j}^{-}$in the derivatives, and that is precisely what we have in (5.7).

${ }^{9}$ The subscript on operators denotes their bare scaling dimensions.
} 
The Fourier transform of this operator gives in momentum space the following:

$$
\begin{aligned}
\widetilde{D}_{1}= & \frac{1}{2}\left\{\epsilon ^ { i j k } \left[z_{1 i} K_{12 k}^{-}\left(\left(-\Delta_{2}+d+k_{2} \cdot \frac{\partial}{\partial k_{2}}\right) k_{1 j}-\left(-\Delta_{1}+d+k_{1} \cdot \frac{\partial}{\partial k_{1}}\right) k_{2 j}\right)\right.\right. \\
& \left.\left.+\left(z_{1} \cdot \frac{\partial}{\partial k_{1}}\right) K_{12 k}^{-} k_{1 i} k_{2 j}-\frac{K_{12}^{-} \cdot K_{12}^{+}}{2} z_{1 k} k_{1 i} k_{2 j}\right]\right\} \\
+ & \frac{1}{2}\left\{\epsilon^{i j k}\left[\left(z_{1} \cdot \frac{\partial}{\partial k_{1}}\right) z_{2 j} \frac{\partial}{\partial z_{2}^{k}}+\left(z_{2} \cdot \frac{\partial}{\partial k_{2}}\right) z_{1 k} \frac{\partial}{\partial z_{2}^{j}}+z_{1 j} z_{2 k}\left(\frac{\partial}{\partial k_{2}} \cdot \frac{\partial}{\partial z_{2}}\right)\right] k_{1 i}\right\} \\
& -\frac{1}{2} \epsilon^{i j k}\left[z_{1 i} z_{2 j} \frac{\partial}{\partial z^{2 k}}\right]\left(-\Delta_{1}+d+k_{1} \cdot \frac{\partial}{\partial k_{1}}\right)
\end{aligned}
$$

The above can be written in a translation invariant manner using the methods implemented in the case of $\widetilde{D}_{12}$ to obtain:

$$
\begin{gathered}
\widetilde{D}_{1}=\left(2-\Delta_{2}\right) \epsilon\left(z_{1} k_{1} K_{12}^{-}\right)-\left(2-\Delta_{1}\right) \epsilon\left(z_{1} k_{2} K_{12}^{-}\right)+\left(z_{1} \cdot K_{12}^{-}\right) \epsilon\left(k_{1} k_{2} K_{12}^{-}\right)-\epsilon\left(z_{1} k_{2} K_{12}^{-}\right)\left(k_{1} \cdot K_{12}^{-}\right) \\
-\epsilon\left(z_{1} k_{1} k_{2}\right) \mathcal{W}_{12}^{--}-\epsilon^{i j k}\left[\left(\left(z_{2} \cdot K_{23}^{-}\right) z_{1 k} \frac{\partial}{\partial z_{2}^{j}}+z_{1 j} z_{2 k}\left(\frac{\partial}{\partial z_{2}} \cdot K_{12}^{-}\right)\right) k_{1 i}\right. \\
\left.+z_{1 i} z_{2 j} \frac{\partial}{\partial z^{2 k}}\left(-\Delta_{1}+d\right)\right]
\end{gathered}
$$

We also introduce the following parity-odd operator:

$$
\begin{aligned}
D_{123} & =\left(X_{i} \cdot X_{j}\right) \epsilon\left(Z_{1} Z_{2} X_{1} X_{2} X_{3}\right) \\
& =\frac{x_{i j}^{2}}{2}\left[2\left(z_{2} \cdot x_{21}\right) \epsilon\left(z_{1} x_{21} x_{31}\right)+x_{12}^{2} \epsilon\left(z_{1} x_{31} z_{2}\right)+x_{31}^{2} \epsilon\left(z_{1} z_{2} x_{21}\right)\right] \quad i, j=1,2,3
\end{aligned}
$$

which after a Fourier transform takes the form:

$$
D_{123}=2\left[\left(z_{2} \cdot K_{21}^{-}\right) \epsilon\left(z_{1} K_{21}^{-} K_{31}^{-}\right)+\epsilon\left(z_{1} K_{31}^{-} z_{2}\right) \mathcal{W}_{12}^{--}+\epsilon\left(z_{1} z_{2} K_{21}^{-}\right) \mathcal{W}_{13}^{--}\right] \mathcal{W}_{i j}^{--}
$$

This operator is naturally translational invariant.

Finally, the polarization vectors can be stripped off from the correlators using the Todorov operator [88]:

$$
D_{z}^{\mu}=\left(\frac{1}{2}+\vec{z} \cdot \frac{\partial}{\partial \vec{z}}\right) \frac{\partial}{\partial z_{\mu}}-\frac{1}{2} z^{\mu} \frac{\partial^{2}}{\partial \vec{z} \cdot \partial \vec{z}} .
$$

We will now discuss the computation of $\langle J J O\rangle_{\text {odd }}$ using the technique of spin-raising and weight-shifting operators.

\subsection{Reproducing $\langle J J O\rangle_{\text {odd }}$ correlators}

We start with the seed correlator $\left\langle\mathrm{O}_{2} \mathrm{O}_{3} \mathrm{O}_{\Delta}\right\rangle$, where the last operator has an arbitrary scaling dimension $\Delta$. To get $\left\langle J J O_{\Delta}\right\rangle_{\text {odd }}$ we act on the scalar-seed with the parity-odd operator $\widetilde{D}_{12}$ defined in (5.7):

$$
\left\langle J^{\mu}\left(k_{1}\right) J^{\nu}\left(k_{2}\right) O_{\Delta}\left(k_{3}\right)\right\rangle_{\text {odd }}=D_{z_{2}}^{\nu} D_{z_{1}}^{\mu} \widetilde{D}_{12}\left\langle O_{2}\left(k_{1}\right) O_{3}\left(k_{2}\right) O_{\Delta}\left(k_{3}\right)\right\rangle
$$




\subsection{1 $\left\langle J^{\mu} J^{\nu} O_{2}\right\rangle_{\text {odd }}$}

Let us consider the case when $\Delta=2$. We have:

$$
\left\langle O_{2}\left(k_{1}\right) O_{3}\left(k_{2}\right) O_{2}\left(k_{3}\right)\right\rangle=c_{1} \log \left(\frac{k_{1}+k_{2}+k_{3}}{\mu}\right)\left(k_{1}+k_{3}\right)-c_{1}\left(k_{1}+k_{2}+k_{3}\right)
$$

Acting with $\widetilde{D}_{12}$ and then removing the polarization vectors gives

$$
\left\langle J^{\mu}\left(k_{1}\right) J^{\nu}\left(k_{2}\right) O_{2}\left(k_{3}\right)\right\rangle_{\text {odd }}=c_{1} \frac{\epsilon^{\mu k_{1} k_{2}}\left(k_{1} k_{2}^{\nu}-k_{2} k_{1}^{\nu}\right)}{k_{1} k_{2}\left(k_{1}+k_{2}+k_{3}\right)^{2}}+c_{1} \frac{\epsilon^{\mu \nu k_{1}}\left(k_{1}+k_{2}-k_{3}\right)}{2 k_{1}\left(k_{1}+k_{2}+k_{3}\right)}
$$

This matches the answer in (4.12). To see this explicitly, we substitute the solution to the form factors given in (4.24) and (4.27) in the ansatz (4.12):

$$
\left\langle J^{\mu}\left(k_{1}\right) J^{\nu}\left(k_{2}\right) O\left(k_{3}\right)\right\rangle_{\text {odd }}=c_{1} \frac{\epsilon^{\nu k_{1} k_{2}}\left(k_{2} k_{1}^{\mu}-k_{1} k_{2}^{\mu}\right)}{k_{1} k_{2}\left(k_{1}+k_{2}+k_{3}\right)^{2}}+c_{1} \frac{\epsilon^{\mu \nu k_{2}}\left(k_{1}+k_{2}-k_{3}\right)}{2 k_{2}\left(k_{1}+k_{2}+k_{3}\right)}
$$

Using the Schouten identities in (C.3) and (C.4) to replace $\epsilon^{\nu k_{1} k_{2}}$ in the above equation, we match the result obtained using weight-shifting and spin-raising operators in (5.16). Similarly, one can obtain and match the results for $\left\langle J J O_{1}\right\rangle_{\text {odd }}$ and $\left\langle J J O_{3}\right\rangle_{\text {odd }}$.

We were also able to obtain $\left\langle J J O_{\Delta}\right\rangle$ for $\Delta=1,2,3$ using the operator in (5.9). However, for $\Delta=3$ we obtained the correlator up to a conformally invariant contact term given by $\epsilon\left(z_{1} z_{2} k_{1}\right)$.

When the dimension of the scalar operator is greater than or equal to 4 , one needs to be more careful as the correlators are divergent and need to be renormalised (see section (4.2.1)).

\subsection{2 $\left\langle J^{\mu} J^{\nu} O_{4}\right\rangle_{\text {odd }}$}

The full seed correlator along with the divergent part is given by:

$$
\begin{aligned}
\left\langle O_{2}\left(k_{1}\right) O_{3}\left(k_{2}\right) O_{4}\left(k_{3}\right)\right\rangle=\frac{c_{1}}{24}[ & -11 k_{1}^{3}-6 k_{1}^{2}\left(k_{2}+k_{3}\right)+3 k_{1}\left(7 k_{2}^{2}+2 k_{2} k_{3}+3 k_{3}^{2}\right) \\
& +4\left(4 k_{2}^{3}+3 k_{2}^{2} k_{3}+k_{3}^{3}\right) \\
& \left.+6 \log \left(\frac{k_{1}+k_{2}+k_{3}}{\mu}\right)\left(k_{1}^{3}-2 k_{2}^{3}-k_{1}\left(3 k_{2}^{2}+k_{3}^{2}\right)\right)\right] \\
& -c_{1} \frac{1}{\epsilon}\left(k_{1}^{3}-2 k_{2}^{3}-k_{1}\left(3 k_{2}^{2}+k_{3}^{2}\right)\right)
\end{aligned}
$$

This correlator has two semi-local divergences labelled by $(+--)$ and $(-+-)$. The generating functional is given by:

$$
Z=\int D \phi \exp \left(-\int d^{3} x\left(S\left[A_{\mu}, g^{\mu \nu}\right]+\sqrt{g}\left(O_{2} \phi_{1}+O_{3} \phi_{0}+O_{4} \phi_{-1}\right)+S_{\mathrm{ct}}\right)\right)
$$

where $\phi_{0}, \phi_{1}$ and $\phi_{-1}$ correspond to the sources of $O_{3}, O_{2}$ and $O_{4}$ respectively. The counter-term in this case is given by

$S_{\mathrm{ct}}=\int d^{3+\epsilon} x \mu^{-\epsilon}\left(a_{1}(\epsilon) \square O_{2} \phi_{0} \phi_{-1}+a_{2}(\epsilon) O_{2} \square \phi_{0} \phi_{-1}+a_{3}(\epsilon) O_{2} \phi_{0} \square \phi_{-1}+a_{4}(\epsilon) O_{3} \phi_{1} \phi_{-1}\right)$ 
The full renormalized correlator is then defined by

$$
\begin{aligned}
&\left\langle O_{2}\left(k_{1}\right) O_{3}\left(k_{2}\right) O_{4}\left(k_{3}\right)\right\rangle_{\text {ren }}= \frac{-8}{\sqrt{g\left(x_{1}\right)} \sqrt{g\left(x_{2}\right)} \sqrt{g\left(x_{3}\right)}} \frac{\delta}{\delta \phi_{1}\left(x_{1}\right)} \frac{\delta}{\delta \phi_{0}\left(x_{2}\right)} \frac{\delta}{\delta \phi_{-1}\left(x_{3}\right)} Z \\
&=c_{1} \frac{1}{24}\left[-11 k_{1}^{3}-6 k_{1}^{2}\left(k_{2}+k_{3}\right)+3 k_{1}\left(7 k_{2}^{2}+2 k_{2} k_{3}+3 k_{3}^{2}\right)\right. \\
&+4\left(4 k_{2}^{3}+3 k_{2}^{2} k_{3}+k_{3}^{3}\right) \\
&\left.+6 \log \left(\frac{k_{1}+k_{2}+k_{3}}{\mu}\right)\left(k_{1}^{3}-2 k_{2}^{3}-k_{1}\left(3 k_{2}^{2}+k_{3}^{2}\right)\right)\right]
\end{aligned}
$$

We now act $\widetilde{D}_{12}$ from (5.7) on the above renormalised scalar correlator and then strip off the polarization vectors to obtain:

$$
\left\langle J^{\mu}\left(k_{1}\right) J^{\nu}\left(k_{2}\right) O_{4}\left(k_{3}\right)\right\rangle_{\text {odd }}=-\epsilon^{k_{1} k_{2} \mu} k_{1}^{\nu} A_{1}+\epsilon^{k_{1} k_{2} \mu} k_{2}^{\nu} A_{2}+\epsilon^{k_{1} \mu \nu}\left(A_{1} k_{1} \cdot k_{2}+A_{2} k_{2}^{2}\right)
$$

where $A_{1}$ is as in (4.36) and $A_{2}\left(k_{1}, k_{2}, k_{3}\right)=-A_{1}\left(k_{2}, k_{1}, k_{3}\right)$. Once again, using the Schouten identities in (C.3) and (C.4), this matches the solution (4.33) and (4.36) obtained by solving the conformal Ward identities.

\subsection{3 $\left\langle J^{\mu} J^{\nu} O_{5}\right\rangle_{\text {odd }}$}

The seed correlator here has ultra-local and non-local divergences. The correlator is given by

$$
\left\langle O_{2}\left(k_{1}\right) O_{3}\left(k_{2}\right) O_{5}\left(k_{3}\right)\right\rangle=\frac{c_{1}}{16 \epsilon}\left(5 k_{1}^{4}-40 k_{1} k_{2}^{3}-15 k_{2}^{4}+6 k_{2}^{2} k_{3}^{2}+k_{3}^{4}-6 k_{1}^{2}\left(5 k_{2}^{2}+k_{3}^{2}\right)\right)+\mathcal{O}\left(\epsilon^{0}\right)
$$

Unlike the previous case, this divergence is removed by taking the constant $c_{1}$ to be $\mathcal{O}(\epsilon)$ and then taking the limit $\epsilon \rightarrow 0$ (similar to (4.39) and (4.40)). Thus we have the renormalised correlator:

$$
\left\langle O_{2}\left(k_{1}\right) O_{3}\left(k_{2}\right) O_{5}\left(k_{3}\right)\right\rangle_{\mathrm{ren}}=c_{1} \frac{1}{16}\left(5 k_{1}^{4}-40 k_{1} k_{2}^{3}-15 k_{2}^{4}+6 k_{2}^{2} k_{3}^{2}+k_{3}^{4}-6 k_{1}^{2}\left(5 k_{2}^{2}+k_{3}^{2}\right)\right)
$$

The rest of the calculation is the same as in the case of $\left\langle J J O_{4}\right\rangle_{\text {odd }}$ and we get

$$
\left\langle J^{\mu}\left(k_{1}\right) J^{\nu}\left(k_{2}\right) O_{5}\left(k_{3}\right)\right\rangle_{\text {odd }}=-\epsilon^{k_{1} k_{2} \mu} k_{1}^{\nu} A_{1}+\epsilon^{k_{1} k_{2} \mu} k_{2}^{\nu} A_{2}+\epsilon^{k_{1} \mu \nu}\left(A_{1} k_{1} \cdot k_{2}+A_{2} k_{2}^{2}\right)
$$

where $A_{1}$ is as in (4.40) and $A_{2}=-A_{1}\left(k_{1} \leftrightarrow k_{2}\right)$. It can be easily checked that this matches the answer obtained by solving conformal Ward identities (see (4.33) and (4.40)).

\section{$6\langle J J J\rangle_{\text {odd }}$ in three-dimensions}

We now turn our attention to computing the odd part of the $\langle J J J\rangle$ correlator. The correlator is non-zero only when the currents are non-Abelian. 
We express $\langle J J J\rangle_{\text {odd }}$ in terms of transverse and longitudinal parts [2]:

$$
\begin{aligned}
\left\langle J^{\mu a} J^{\nu b} J^{\rho c}\right\rangle_{\text {odd }}=\left\langle j^{\mu a} j^{\nu b} j^{\rho c}\right\rangle_{\text {odd }}+ & {\left[\frac{k_{1}^{\mu}}{k_{1}^{2}}\left(f^{a d c}\left\langle J^{\rho d}\left(k_{2}\right) J^{\nu b}\left(-k_{2}\right)\right\rangle-f^{a b d}\left\langle J^{\nu d}\left(k_{3}\right) J^{\rho c}\left(-k_{3}\right)\right\rangle\right)\right.} \\
& \left.+\left(\left(k_{1}, \mu\right) \leftrightarrow\left(k_{2}, \nu\right)\right)+\left(\left(k_{1}, \mu\right) \leftrightarrow\left(k_{3}, \rho\right)\right)\right] \\
+ & {\left[\left(\frac{k_{1}^{\mu} k_{2}^{\nu}}{k_{1}^{2} k_{2}^{2}} f^{a b d} k_{2 \alpha}\left\langle J^{\alpha d}\left(k_{3}\right) J^{\rho c}\left(-k_{3}\right)\right\rangle\right)\right.} \\
& \left.+\left(\left(k_{1}, \mu\right) \leftrightarrow\left(k_{3}, \rho\right)\right)+\left(\left(k_{2}, \nu\right) \leftrightarrow\left(k_{3}, \rho\right)\right)\right]
\end{aligned}
$$

where $\left\langle j^{\mu a} j^{\nu b} j^{\rho c}\right\rangle_{\text {odd }}$ denotes the transverse part of the correlator. The ansatz for this part of the correlator can be written as

$$
\left\langle j^{\mu a} j^{\nu b} j^{\rho c}\right\rangle_{\text {odd }}=\pi_{\alpha}^{\mu}\left(k_{1}\right) \pi_{\beta}^{\nu}\left(k_{2}\right) \pi_{\gamma}^{\rho}\left(k_{3}\right) X^{\alpha \beta \gamma}
$$

where

$$
X^{\alpha \beta \gamma}=A_{1} \epsilon^{k_{1} k_{2} \alpha} k_{1}^{\gamma} k_{3}^{\beta}+A_{2} \epsilon^{k_{1} k_{2} \alpha} \delta^{\beta \gamma}+A_{3} \epsilon^{k_{1} \alpha \beta} k_{1}^{\gamma}+A_{4} \epsilon^{k_{1} \alpha \gamma} k_{3}^{\beta}+\text { cyclic perm. }
$$

Calculating the form factors by directly solving the conformal Ward identities is quite complicated. Here we will instead use spin-raising and weight shifting operators to calculate them.

Starting from the seed correlator $\left\langle O_{3}\left(k_{1}\right) O_{2}\left(k_{2}\right) O_{3}\left(k_{3}\right)\right\rangle$ we can get $\langle J J J\rangle_{\text {odd }}$ by:

$\left\langle J^{\mu a}\left(k_{1}\right) J^{\nu b}\left(k_{2}\right) J^{\rho c}\left(k_{3}\right)\right\rangle_{\text {odd }}=\frac{1}{4} D_{z_{1}}^{\mu} D_{z_{2}}^{\nu} D_{z_{3}}^{\rho} D_{11} \widetilde{D}_{23}\left\langle O_{3}^{a}\left(k_{1}\right) O_{2}^{b}\left(k_{2}\right) O_{3}^{c}\left(k_{3}\right)\right\rangle_{\text {even }}+$ cyclic perm.

where $\widetilde{D}_{12}$ is defined in (5.7) and $D_{11}$ in (E.5). The renormalized seed correlator is:

$$
\begin{aligned}
\left\langle O_{3}^{a}\left(k_{1}\right) O_{2}^{b}\left(k_{2}\right) O_{3}^{c}\left(k_{3}\right)\right\rangle=f^{a b c} c_{1}[ & 2 \log \left(\frac{k_{1}+k_{2}+k_{3}}{\mu}\right)\left(k_{1}^{2}-k_{2}^{2}+k_{3}^{2}\right)-k_{1}^{2}-k_{3}^{2} \\
& \left.+2 k_{1}\left(k_{2}-k_{3}\right)+2 k_{2} k_{3}+3 k_{2}^{2}\right]
\end{aligned}
$$

This gives:

$$
\begin{aligned}
X^{\alpha \beta \gamma}= & -\frac{2}{k_{1}\left(k_{1}+k_{2}+k_{3}\right)^{3}} \epsilon^{k_{1} k_{2} \alpha} k_{1}^{\gamma} k_{3}^{\beta}-\frac{1}{\left(k_{1}+k_{2}+k_{3}\right)^{2}} \epsilon^{k_{1} k_{2} \alpha} \delta^{\beta \gamma} \\
& +\frac{\left(k_{1}+k_{2}+2 k_{3}\right)}{k_{1}\left(k_{1}+k_{2}+k_{3}\right)^{2}} \epsilon^{k_{1} \alpha \beta} k_{1}^{\gamma}+\frac{\left(k_{1}+2 k_{2}+k_{3}\right)}{k_{1}\left(k_{1}+k_{2}+k_{3}\right)^{2}} \epsilon^{k_{1} \alpha \gamma} k_{3}^{\beta} \\
& + \text { cyclic perm. }
\end{aligned}
$$

We can now read off the form factors in (6.3) by comparing it with (6.6):

$$
\begin{aligned}
A_{1} & =-\frac{2}{k_{1}\left(k_{1}+k_{2}+k_{3}\right)^{3}}, & A_{2} & =-\frac{1}{\left(k_{1}+k_{2}+k_{3}\right)^{2}} \\
A_{3} & =\frac{k_{1}+k_{2}+2 k_{3}}{k_{1}\left(k_{1}+k_{2}+k_{3}\right)^{2}}, & A_{4} & =\frac{k_{1}+2 k_{2}+k_{3}}{k_{1}\left(k_{1}+k_{2}+k_{3}\right)^{2}}
\end{aligned}
$$


The $\langle J J J\rangle_{\text {odd }}$ correlator obeys the following Ward-Takahashi identity [2]:

$$
\begin{aligned}
k_{1 \mu}\left\langle J^{\mu a}\left(k_{1}\right) J^{\nu b}\left(k_{2}\right) J^{\rho c}\left(k_{3}\right)\right\rangle_{\text {odd }} & =f^{a d c}\left\langle J^{\rho d}\left(k_{2}\right) J^{\nu b}\left(-k_{2}\right)\right\rangle_{\text {odd }}-f^{a b d}\left\langle J^{\nu d}\left(k_{3}\right) J^{\rho c}\left(-k_{3}\right)\right\rangle_{\text {odd }} \\
& =-f^{a b c}\left(\epsilon^{k_{2} \nu \rho}+\epsilon^{k_{3} \nu \rho}\right)
\end{aligned}
$$

Our result does satisfy this identity. To see this, let us contract our result (6.1) for $\left\langle J^{\mu a} J^{\nu b} J^{\rho c}\right\rangle$ with $k_{1}^{\mu}$ :

$$
\begin{aligned}
k_{1 \mu}\left\langle J^{\mu a} J^{\nu b} J^{\rho c}\right\rangle_{\text {odd }}= & f^{a b c} \epsilon^{k_{1} k_{2} \nu}\left(B_{1} k_{1}^{\rho}+\left(B_{1}-B_{1}\left(k_{2} \leftrightarrow k_{3}\right)\right) k_{3}^{\rho}\right) \\
& +f^{a b c} \epsilon^{k_{1} k_{2} \rho}\left(B_{1} k_{2}^{\nu}+B_{1}\left(k_{2} \leftrightarrow k_{3}\right) k_{3}^{\nu}\right)-2\left(\epsilon^{k_{2} \nu \rho} B_{2}-\epsilon^{k_{3} \nu \rho} B_{2}\left(k_{2} \leftrightarrow k_{3}\right)\right)
\end{aligned}
$$

where

$$
B_{1}=\frac{4\left(2 k_{1}+k_{2}+k_{3}\right)}{k_{2}\left(k_{1}+k_{2}+k_{3}\right)^{2}}, \quad B_{2}=\frac{2 k_{1}^{2}+k_{2}^{2}+k_{1}\left(k_{2}-k_{3}\right)-k_{3}^{2}}{k_{2}\left(k_{1}+k_{2}+k_{3}\right)}
$$

Using the identities in (C.6) and (C.7), we can get rid of those epsilon structures which have two momenta contracted with their indices. Doing so we obtain:

$$
k_{1 \mu}\left\langle J^{\mu a} J^{\nu b} J^{\rho c}\right\rangle=-f^{a b c}\left(\epsilon^{k_{2} \nu \rho}+\epsilon^{k_{3} \nu \rho}\right)
$$

which matches the desired Ward identity (6.8).

\section{$7\langle T T O\rangle_{\text {odd }}$ in three-dimensions}

We now turn our attention to the $\langle T T O\rangle_{\text {odd }}$ correlator. Based on symmetry considerations and conservation, this correlator is expected to take the following form [2]:

$$
\begin{aligned}
& \left\langle T^{\mu_{1} \nu_{1}}\left(k_{1}\right) T^{\mu_{2} \nu_{2}}\left(k_{2}\right) O^{I}\left(k_{3}\right)\right\rangle_{\text {odd }}=\left\langle t^{\mu_{1} \nu_{1}}\left(k_{1}\right) t^{\mu_{2} \nu_{2}}\left(k_{2}\right) O^{I}\left(k_{3}\right)\right\rangle_{\text {odd }} \\
& \quad+2\left[\mathcal{T}^{\mu_{1} \nu_{1} \alpha_{1}}\left(k_{1}\right) k_{1}^{\beta_{1}}+\frac{\pi^{\mu_{1} \nu_{1}}\left(k_{1}\right)}{d-1} \delta^{\alpha_{1} \beta_{1}}\right] \delta^{\mu_{2} \alpha_{2}} \delta^{\nu_{2} \beta_{2}}\left\langle\frac{\delta T_{\alpha_{1} \beta_{1}}}{\delta g^{\alpha_{2} \beta_{2}}}\left(k_{1}, k_{2}\right) O^{I}\left(k_{3}\right)\right\rangle \\
& +2\left[\left(\mu_{1}, \nu_{1}, k_{1}\right) \leftrightarrow\left(\mu_{2}, \nu_{2}, k_{2}\right)\right] \\
& -4\left[\mathcal{T}^{\mu_{1} \nu_{1} \alpha_{1}}\left(k_{1}\right) k_{1}^{\beta_{1}}+\frac{\pi^{\mu_{1} \nu_{1}}\left(k_{1}\right)}{d-1} \delta^{\alpha_{1} \beta_{1}}\right]\left[\mathcal{T}^{\mu_{2} \nu_{2} \alpha_{2}}\left(k_{2}\right) k_{2}^{\beta_{2}}+\frac{\pi^{\mu_{2} \nu_{2}}\left(k_{2}\right)}{d-1} \delta^{\alpha_{2} \beta_{2}}\right] \\
& \quad \times\left\langle\frac{\delta T_{\alpha_{1} \beta_{1}}}{\delta g^{\alpha_{2} \beta_{2}}}\left(k_{1}, k_{2}\right) O^{I}\left(k_{3}\right)\right\rangle
\end{aligned}
$$

where

$$
\mathcal{T}^{\mu \nu \alpha}(p)=\eta^{\alpha \beta} \mathcal{T}_{\beta}^{\mu \nu}=\frac{\eta^{\alpha \beta}}{p^{2}}\left[2 p^{(\mu} \delta_{\beta}^{\nu)}-\frac{p_{\beta}}{d-1}\left(\delta^{\mu \nu}+(d-2) \frac{p^{\mu} p^{\nu}}{p^{2}}\right)\right]
$$

and $\left\langle t^{\mu_{1} \nu_{1}}\left(k_{1}\right) t^{\mu_{2} \nu_{2}}\left(k_{2}\right) O^{I}\left(k_{3}\right)\right\rangle_{\text {odd }}$ is the transverse part of the correlator. Due to symmetry and transversality, this is expected to take the following form:

$$
\begin{aligned}
& \left\langle t^{\mu_{1} \nu_{1}}\left(k_{1}\right) t^{\mu_{2} \nu_{2}}\left(k_{2}\right) O^{I}\left(k_{3}\right)\right\rangle_{\text {odd }}= \\
& =\Pi_{\alpha_{1} \beta_{1}}^{\mu_{1} \nu_{1}}\left(k_{1}\right) \Pi_{\alpha_{2} \beta_{2}}^{\mu_{2} \nu_{2}}\left(k_{2}\right)[ \\
& A_{11} \epsilon^{\alpha_{1} k_{1} k_{2}} k_{2}^{\beta_{1}} k_{1}^{\beta_{2}} k_{1}^{\alpha_{2}}+A_{12} \epsilon^{\alpha_{2} k_{1} k_{2}} k_{2}^{\beta_{1}} k_{1}^{\beta_{2}} k_{2}^{\alpha_{1}}+A_{21} \epsilon^{\alpha_{1} k_{1} k_{2}} \delta^{\beta_{1} \beta_{2}} k_{1}^{\alpha_{2}} \\
& \quad+A_{22} \epsilon^{\alpha_{2} k_{1} k_{2}} \delta^{\beta_{1} \beta_{2}} k_{2}^{\alpha_{1}}+A_{31} \epsilon^{\alpha_{1} \alpha_{2} k_{1}} k_{2}^{\beta_{1}} k_{1}^{\beta_{2}}+A_{32} \epsilon^{\alpha_{1} \alpha_{2} k_{2}} k_{2}^{\beta_{1}} k_{1}^{\beta_{2}} \\
& \left.+A_{41} \epsilon^{\alpha_{1} \alpha_{2} k_{1}} \delta^{\beta_{1} \beta_{2}}+A_{42} \epsilon^{\alpha_{1} \alpha_{2} k_{2}} \delta^{\beta_{1} \beta_{2}}\right] .
\end{aligned}
$$


where the traceless-orthogonal projector is given by:

$$
\Pi_{\alpha \beta}^{\mu \nu}(p) \equiv \frac{1}{2}\left(\pi_{\alpha}^{\mu}(p) \pi_{\beta}^{\nu}(p)+\pi_{\beta}^{\mu}(p) \pi_{\alpha}^{\nu}(p)\right)-\frac{1}{2} \pi^{\mu \nu}(p) \pi_{\alpha \beta}(p)
$$

In the following, we first present a direct calculation of $\langle T T O\rangle$ in the free-fermion theory for which $O=\bar{\psi} \psi$ which is parity-odd in three-dimensions and has scaling dimension $\Delta=2$. We then reproduce the free theory answer using spin and weight-shifting operators. We also compute $\langle T T O\rangle_{\text {odd }}$ with $\Delta_{O}=1$ which does not have a free theory analogue. ${ }^{10} \mathrm{We}$ defer the computation of $\left\langle T T O_{\Delta}\right\rangle$ for general $\Delta$ to future work.

\section{$7.1\left\langle\mathrm{TTO}_{2}\right\rangle$ for free fermionic theory}

The free-fermion action in curved space is given by [2]:

$$
S=\int d^{3} x e\left[\bar{\psi} e_{a}^{\mu} \gamma^{a} \stackrel{\leftrightarrow}{\nabla}_{\mu} \psi\right]
$$

where $e_{\mu}^{a}$ are the vielbeins and the covariant derivative acts as follows on the spinor

$$
\begin{aligned}
\nabla_{\mu} \psi & =\left(\partial_{\mu}-\frac{i}{2} \omega_{\mu}^{a b} \Sigma_{a b}\right) \psi \\
\bar{\psi} \overleftarrow{\nabla}_{\mu} & =\bar{\psi}\left(\overleftarrow{\partial}_{\mu}+\frac{i}{2} \omega_{\mu}^{a b} \Sigma_{a b}\right)
\end{aligned}
$$

One may use this action to compute the stress-energy tensor [2]:

$$
T_{\mu \nu}=\frac{1}{\sqrt{g}} \frac{\delta S}{\delta g^{\mu \nu}}=\frac{1}{2} \bar{\psi} \gamma_{(\mu} \overleftrightarrow{\nabla}_{\nu)} \psi
$$

which after taking the flat-space limit and a Fourier transform gives the stress-energy tensor in momentum space:

$$
T_{\mu \nu}(k)=\frac{1}{4} \int d^{3} l \bar{\psi}(l)\left[\gamma_{\mu}(2 l-k)_{\nu}+\gamma_{\nu}(2 l-k)_{\mu}\right] \psi(k-l)
$$

The parity-odd scalar primary in the free-fermion theory is given by:

$$
O_{2}=\bar{\psi} \psi
$$

Using these definitions it is straightforward to evaluate $\left\langle T T \mathrm{O}_{2}\right\rangle$ for the free-fermionic theory. Since $\mathrm{O}_{2}$ is parity-odd $\left\langle T T \mathrm{O}_{2}\right\rangle$ is also parity-odd. We give the details of the computation in appendix D and give only the final results here. The form factors that appear in

\footnotetext{
${ }^{10}$ One can get such a parity-odd correlator by coupling a complex scalar field theory to a Chern-Simons gauge field. See [71-75].
} 
the transverse part of the correlator (7.3) are:

$$
\begin{aligned}
& A_{11}=\frac{k_{1}+4 k_{2}+k_{3}}{6\left(k_{1}+k_{2}+k_{3}\right)^{4}} \\
& A_{21}=\frac{2 k_{1}^{2}+4 k_{2}^{2}+3 k_{2} k_{3}+k_{3}^{2}+3 k_{1}\left(2 k_{2}+k_{3}\right)}{6\left(k_{1}+k_{2}+k_{3}\right)^{3}} \\
& A_{31}=\frac{k_{3}\left(k_{1}+3 k_{2}+k_{3}\right)}{4\left(k_{1}+k_{2}+k_{3}\right)^{3}} \\
& A_{41}=-\frac{k_{1}^{3}+2 k_{1}^{2}\left(k_{2}+k_{3}\right)+2 k_{3}^{2}\left(2 k_{2}+k_{3}\right)+k_{1}\left(k_{2}^{2}+2 k_{2} k_{3}+3 k_{3}^{2}\right)}{8\left(k_{1}+k_{2}+k_{3}\right)^{2}} \\
& A_{i 2}=-A_{i 1}\left(k_{1} \leftrightarrow k_{2}\right) .
\end{aligned}
$$

To compute the longitudinal part, we require the functional derivative of the fermionic stress-energy tensor. Using the action of the covariant derivative on spinors (7.6) we reexpress the stress-energy tensor (7.7) as:

$$
T_{\mu \nu}=\frac{1}{2} \bar{\psi} \gamma_{(\mu} \stackrel{\leftrightarrow}{\partial}_{\nu)} \psi+\frac{1}{16} \omega_{(\mu}^{a b} \bar{\psi}\left\{\gamma_{\nu)}, \gamma_{a b}\right\} \psi
$$

After some computation (see appendix D.2) we obtain:

$$
\frac{\delta T_{\mu \nu}(x)}{\delta g_{\alpha \beta}(y)}=-\frac{i}{16}\left\{\left[\epsilon_{\sigma \alpha \mu} \delta_{\beta \nu}+\epsilon_{\sigma \beta \mu} \delta_{\alpha \nu}+\epsilon_{\sigma \alpha \nu} \delta_{\beta \mu}+\epsilon_{\sigma \beta \nu} \delta_{\alpha \mu}\right] \partial^{\sigma} \delta^{(3)}(x-y)\right\} O_{2}(x)
$$

Taking a Fourier transform of the above we get:

$$
\frac{\delta T_{\mu \nu}}{\delta g_{\alpha \beta}}\left(k_{1}, k_{2}\right)=\frac{1}{32}\left[\epsilon_{k_{2} \alpha \mu} \delta_{\beta \nu}+\epsilon_{k_{2} \beta \mu} \delta_{\alpha \nu}+\epsilon_{k_{2} \alpha \nu} \delta_{\beta \mu}+\epsilon_{k_{2} \beta \nu} \delta_{\alpha \mu}\right] O_{2}\left(k_{3}\right)-\left(k_{1} \leftrightarrow k_{2}\right)
$$

Using the above, one may compute

$$
\left\langle\frac{\delta T_{\mu \nu}}{\delta g_{\alpha \beta}}\left(k_{1}, k_{2}\right) O_{2}\left(-k_{3}\right)\right\rangle=-\frac{k_{3}}{256}\left[\epsilon_{k_{2} \alpha \mu} \delta_{\beta \nu}+\epsilon_{k_{2} \beta \mu} \delta_{\alpha \nu}+\epsilon_{k_{2} \alpha \nu} \delta_{\beta \mu}+\epsilon_{k_{2} \beta \nu} \delta_{\alpha \mu}-\left(k_{1} \leftrightarrow k_{2}\right)\right]
$$

where we used $\left\langle O_{2}\left(k_{3}\right) O_{2}\left(-k_{3}\right)\right\rangle=-\frac{k_{3}}{8}$ in the free-fermion theory. This can now be used in the reconstruction formula 7.1 to get the full correlator $\left\langle T T \mathrm{O}_{2}\right\rangle$.

Having computed the functional derivative, we now give the trace and transverse Ward identities associated with $\left\langle T T O_{2}\right\rangle$. They are [2]:

$$
\begin{aligned}
\left\langle T\left(k_{1}\right) T_{\alpha \beta}\left(k_{2}\right) O_{2}\left(k_{3}\right)\right\rangle & =2\left\langle\frac{\delta T}{\delta g^{\alpha \beta}}\left(k_{1}, k_{2}\right) O\left(k_{3}\right)\right\rangle \\
k_{1}^{\mu}\left\langle T_{\mu \nu}\left(k_{1}\right) T_{\alpha \beta}\left(k_{2}\right) O_{2}\left(k_{3}\right)\right\rangle & =2 k_{1}^{\mu}\left\langle\frac{\delta T_{\mu \nu}}{\delta g^{\alpha \beta}}\left(k_{1}, k_{2}\right) O\left(k_{3}\right)\right\rangle
\end{aligned}
$$

The expression obtained in (7.14) is traceless in $(\mu, \nu)$ and $(\alpha, \beta)$. This immediately implies that the trace Ward identity is trivial:

$$
\left\langle T\left(k_{1}\right) T_{\alpha \beta}\left(k_{2}\right) O\left(k_{3}\right)\right\rangle=0
$$


Contracting (7.14) with $k_{1}^{\mu}$ gives the transverse Ward identity:

$$
\begin{aligned}
k_{1}^{\mu}\left\langle T_{\mu \nu}\left(k_{1}\right) T_{\alpha \beta}\left(k_{2}\right) O_{2}\left(k_{3}\right)\right\rangle=\frac{k_{3}}{128}[ & -\epsilon_{k_{1} k_{2} \alpha} \delta_{\beta \nu}-\epsilon_{k_{1} k_{2} \beta} \delta_{\alpha \nu}+k_{1 \beta}\left(\epsilon_{k_{1} \alpha \nu}-\epsilon_{k_{2} \alpha \nu}\right) \\
& \left.+k_{1 \alpha}\left(\epsilon_{k_{1} \beta \nu}-\epsilon_{k_{2} \beta \nu}\right)\right]
\end{aligned}
$$

In appendix (D.1) we show that precisely this Ward identity holds.

\section{$7.2\left\langle\mathrm{TTO}_{2}\right\rangle_{\text {odd }}$ using parity-odd spin-raising and weight-shifting operators}

In this section we compute the odd part of $\left\langle T T O_{2}\right\rangle$ using spin-raising and weight-shifting operators. We start from the renormalised scalar-seed correlator $\left\langle O_{1}\left(k_{1}\right) O_{2}\left(k_{2}\right) O_{2}\left(k_{3}\right)\right\rangle$ given by:

$$
\left\langle O_{1}\left(k_{1}\right) O_{2}\left(k_{2}\right) O_{2}\left(k_{3}\right)\right\rangle=\frac{1}{k_{1}} \log \left[\frac{k_{1}+k_{2}+k_{3}}{\mu}\right]
$$

where $\mu$ is the renormalisation scale. We obtain $\left\langle T T O_{2}\right\rangle_{\text {odd }}$ from $\left\langle\mathrm{O}_{1} \mathrm{O}_{2} \mathrm{O}_{2}\right\rangle$ as follows:

$$
\left\langle T T O_{2}\right\rangle_{\text {odd }}=\left(k_{1} k_{2}\right)^{3} P_{1}^{(2)} P_{2}^{(2)} H_{12} \widetilde{D}_{12}\left\langle O_{1}\left(k_{1}\right) O_{2}\left(k_{2}\right) O_{2}\left(k_{3}\right)\right\rangle
$$

After making use of Schouten identities this takes the following explicit form

$$
\left\langle T T O_{2}\right\rangle_{\text {odd }}=k_{1}^{2} k_{2}^{2}\left(k_{2} \cdot z_{1}\right)\left(k_{1} \cdot z_{2}\right) \frac{k_{1}\left(k_{2} \cdot z_{1}\right) \epsilon^{k_{1} k_{2} z_{2}}-k_{2}\left(k_{1} \cdot z_{2}\right) \epsilon^{k_{1} k_{2} z_{1}}}{\left(k_{1}+k_{2}+k_{3}\right)^{4}\left(k_{1}^{2}-2 k_{1} k_{2}+k_{2}^{2}-k_{3}^{2}\right)^{2}}
$$

We can easily check that the expression obtained for the correlator from the free fermion (FF) theory computation in section 7.1 precisely matches the above expression for the correlator up to an additional contact term

$$
\left\langle T^{\mu_{1} \nu_{1}} T^{\mu_{2} \nu_{2}} O_{2}\right\rangle_{\text {odd }}=\left\langle T^{\mu_{1} \nu_{1}} T^{\mu_{2} \nu_{2}} O_{2}\right\rangle_{\mathrm{FF}}+32\left\langle\frac{\delta T^{\mu_{1} \nu_{1}}}{\delta g_{\mu_{2} \nu_{2}}}\left(k_{1}, k_{2}\right) O_{2}\left(-k_{3}\right)\right\rangle
$$

To arrive at (7.22) we have made repeated use of Schouten identities given in appendix C. This additional term can be calculated using (7.14). It can be easily checked going to position space that this additional term is a contact term. This difference can be accounted for through a suitable redefinition of the correlation function as was done in [9], see appendix A.3 of the same paper for more details.

\section{3 $\left\langle T T O_{1}\right\rangle_{\text {odd }}$ using parity-odd spin-raising and weight-shifting operators}

In this subsection we discuss the parity-odd part of the correlator $\left\langle T T O_{1}\right\rangle$. To construct this correlator we start from the regularised scalar seed correlator $\left\langle O_{1}\left(k_{1}\right) O_{2}\left(k_{2}\right) O_{1}\left(k_{3}\right)\right\rangle$ given by:

$$
\left\langle O_{1}\left(k_{1}\right) O_{2}\left(k_{2}\right) O_{1}\left(k_{3}\right)\right\rangle=\frac{1}{k_{1} k_{3} \epsilon}-\frac{1}{k_{1} k_{3}} \log \left[k_{1}+k_{2}+k_{3}\right]
$$

The seed correlator here has a non-local divergence while $\left\langle T T O_{1}\right\rangle$ has no divergence. As noted in section 2.3.1, it would not be possible to reach $\left\langle T T O_{1}\right\rangle$ starting from the renormalized seed correlator in this case. However, if we act with the weight-shifting operators on just the regularised seed correlator, then we see that the divergences cancel in the final 
answer upon using Schouten identities and we get the correct, non-divergent answer for $\left\langle T T O_{1}\right\rangle$. The correlator $\left\langle T T O_{1}\right\rangle$ is obtained by the same operation as in (7.20) now on the seed correlator $\left\langle O_{1}\left(k_{1}\right) O_{2}\left(k_{2}\right) O_{1}\left(k_{3}\right)\right\rangle$ :

$$
\left\langle T T O_{1}\right\rangle_{\text {odd }}=\left(k_{1} k_{2}\right)^{3} P_{1}^{(2)} P_{2}^{(2)} H_{12} \widetilde{D}_{12}\left\langle O_{1}\left(k_{1}\right) O_{2}\left(k_{2}\right) O_{1}\left(k_{3}\right)\right\rangle
$$

From the explicit expression for $\left\langle T T O_{1}\right\rangle_{\text {odd }}$ computed using the above equation one can see that the following relation holds between $\left\langle T T O_{1}\right\rangle_{\text {odd }}$ and $\left\langle T T O_{2}\right\rangle_{\text {odd }}$

$$
\left\langle T T O_{1}\right\rangle_{\text {odd }}=\frac{1}{k_{3}}\left\langle T T O_{2}\right\rangle_{\text {odd }}
$$

This is consistent with the fact that the two scalar operators with $\Delta=1$ and $\Delta=2$ are related by a shadow transformation in three-dimensions.

\section{Momentum space parity-odd 3-point correlators in four-dimensions}

In this section we will construct and use parity-odd spin-raising operators in fourdimensions to determine the non-trivial momentum-space correlator $\langle J J J\rangle$. In fourdimensions, the only non-zero parity-odd 3-point function is $\langle J J J\rangle$ with a nonabelian current $J$. Other correlators involving operators with spin $s \leq 2$ are zero as emphasized in subsection 3.2.

\subsection{Spin-raising and weight-shifting operators}

In four-dimensions, transversality and interiority allow for only one operator:

$$
\begin{aligned}
D_{12}^{++} \equiv & \epsilon \\
=\frac{1}{2} & \left\{-Z_{1} Z_{2} X_{1} X_{2} \frac{\partial}{\partial X_{1}} \frac{\partial}{\partial X_{2}} z_{1 j} z_{2 k} x_{12 l}\left(D_{1} P_{2 i}-D_{2} P_{1 i}\right)\right. \\
& \left.+\epsilon^{i j k l}\left[\left(z_{2} \cdot x_{2}\right) z_{1 k} x_{12 l}-\left(z_{1} \cdot x_{1}\right) z_{2 k} x_{12 l}-\left(\frac{x_{1}^{2}-x_{2}^{2}}{2}\right) z_{1 k} z_{2 l}\right] P_{1 i} P_{2 j}\right\}
\end{aligned}
$$

where the embedding space result has been converted to ordinary position space. Fourier transform of the above operator gives:

$$
\begin{aligned}
D_{12}^{++}=\frac{1}{2}\{ & -\epsilon^{i j k l} z_{1 j} z_{2 k} K_{12 l}^{-}\left[\left(-\Delta_{1}+d+k_{1} \cdot \frac{\partial}{\partial k_{1}}\right) k_{2 i}-\left(-\Delta_{2}+d+k_{2} \cdot \frac{\partial}{\partial k_{2}}\right) k_{1 i}\right] \\
+ & \left.\epsilon^{i j k l}\left[\left(z_{2} \cdot \frac{\partial}{\partial k_{2}}\right) z_{1 k} K_{12 l}^{-}-\left(z_{1} \cdot \frac{\partial}{\partial k_{1}}\right) z_{2 k} K_{12 l}^{-}-\frac{K_{12}^{-} K_{12}^{+}}{2} z_{1 k} z_{2 l}\right] k_{1 i} k_{2 j}\right\}
\end{aligned}
$$

Let us now consider the following commutator:

$$
\begin{aligned}
& {\left[k_{1}^{\mu}+k_{2}^{\mu}+k_{3}^{\mu}, D_{12}^{++}\right]=} \\
& =-\epsilon\left(z_{1} z_{2} K_{12}^{-} k_{2}\right) k_{1}^{\mu}+\epsilon\left(z_{1} z_{2} K_{12}^{-} k_{1}\right) k_{2}^{\mu}-z_{2}^{\mu} \epsilon\left(z_{1} k_{1} k_{2} K_{12}^{-}\right)+z_{1}^{\mu} \epsilon\left(z_{2} k_{1} k_{2} K_{12}^{-}\right)+K_{12}^{-\mu} \epsilon\left(z_{1} z_{2} k_{1} k_{2}\right)
\end{aligned}
$$


which is zero by momentum conservation. The above commutator gives an operator-based Schouten identity. We find the following contracted form of the above equation useful in the present context:

$$
\begin{gathered}
-\epsilon\left(z_{1} z_{2} K_{12}^{-} k_{2}\right) k_{1} \cdot \frac{\partial}{\partial k_{1}}+\epsilon\left(z_{1} z_{2} K_{12}^{-} k_{1}\right) k_{2} \cdot \frac{\partial}{\partial k_{1}}-3 \epsilon\left(z_{1} z_{2} K_{12}^{-} k_{2}\right) \\
-z_{2} \cdot \frac{\partial}{\partial k_{1}} \epsilon\left(z_{1} k_{1} k_{2} K_{12}^{-}\right)+z_{1} \cdot \frac{\partial}{\partial k_{1}} \epsilon\left(z_{2} k_{1} k_{2} K_{12}^{-}\right)+K_{12}^{-} \cdot \frac{\partial}{\partial k_{1}} \epsilon\left(z_{1} z_{2} k_{1} k_{2}\right)=0
\end{gathered}
$$

The above contracted form allows us to write a manifestly translation invariant form of the spin-raising operator:

$$
\begin{aligned}
& D_{12}^{++}=\frac{1}{2}\left\{\epsilon\left(z_{1} z_{2} K_{12}^{-} k_{2}\right)\left(-\Delta_{1}+2\right)-\epsilon\left(z_{1} z_{2} K_{12}^{-} k_{1}\right)\left(-\Delta_{2}+3\right)-\left(z_{2} \cdot K_{12}^{-}\right) \epsilon\left(z_{1} k_{1} k_{2} K_{12}^{-}\right)\right. \\
& \left.+\epsilon\left(z_{1} z_{2} K_{12}^{-} k_{1}\right)\left(k_{2} \cdot K_{12}^{-}\right)+\epsilon\left(z_{1} z_{2} k_{1} k_{2}\right) \mathcal{W}_{12}^{--}\right\}
\end{aligned}
$$

As in (5.7) the operator depends only on $K_{i j}^{-}$in the derivatives, ensuring translational invariance.

\section{$8.2\langle J J J\rangle_{\text {odd }}$}

In this section we use the operator in (8.5) to derive the odd part of $\langle J J J\rangle$. In fourdimensions, this correlator is expected to have the following structure based on transversality and momentum conservation:

$$
\begin{aligned}
\left\langle J_{\mu}^{a}\left(k_{1}\right) J_{\nu}^{b}\left(k_{2}\right) J_{\lambda}^{c}\left(k_{3}\right)\right\rangle_{\text {odd }}=\pi_{\mu}^{\alpha}\left(k_{1}\right) & \pi_{\nu}^{\beta}\left(k_{2}\right) \pi_{\lambda}^{\gamma}\left(k_{3}\right)\left[A^{a b c} \epsilon_{\alpha \beta \gamma k_{1}}+B^{a b c} \epsilon_{\alpha \beta \gamma k_{2}}\right. \\
& \left.+C^{a b c} k_{1 \gamma} \epsilon_{\alpha \beta k_{1} k_{2}}+D^{a b c} k_{2 \alpha} \epsilon_{\beta \gamma k_{1} k_{2}}+E^{a b c} k_{1 \beta} \epsilon_{\gamma \alpha k_{1} k_{2}}\right]
\end{aligned}
$$

The above ansatz can be simplified using the Schouten identities in (C.8) to the following

$$
\begin{aligned}
\left\langle J_{\mu}^{a}\left(k_{1}\right) J_{\nu}^{b}\left(k_{2}\right) J_{\lambda}^{c}\left(k_{3}\right)\right\rangle_{\text {odd }}= & \pi_{\mu}^{\alpha}\left(k_{1}\right) \pi_{\nu}^{\beta}\left(k_{2}\right) \pi_{\lambda}^{\gamma}\left(k_{3}\right) \\
& \times\left[C^{a b c} k_{1 \gamma} \epsilon_{\alpha \beta k_{1} k_{2}}+D^{a b c} k_{2 \alpha} \epsilon_{\beta \gamma k_{1} k_{2}}+E^{a b c} k_{1 \beta} \epsilon_{\gamma \alpha k_{1} k_{2}}\right]
\end{aligned}
$$

Due to cyclic symmetry we obtain the following relations between the form factors

$$
\begin{aligned}
& C^{a b c}\left(k_{1}, k_{2}, k_{3}\right)=-E^{b c a}\left(k_{2}, k_{3}, k_{1}\right) \\
& E^{a b c}\left(k_{1}, k_{2}, k_{3}\right)=-D^{b c a}\left(k_{2}, k_{3}, k_{1}\right) \\
& D^{a b c}\left(k_{1}, k_{2}, k_{3}\right)=-C^{b c a}\left(k_{2}, k_{3}, k_{1}\right)
\end{aligned}
$$

This shows that there is only one independent form factor. To compute $\langle J J J\rangle_{\text {odd }}$ in fourdimensions one has to compute:

$$
\langle J J J\rangle_{\text {odd }}=D_{12}^{++}\left\langle O_{3} O_{3} J\right\rangle+\text { cyclic perms. }
$$

The correlator $\left\langle\mathrm{O}_{3} \mathrm{O}_{3} J\right\rangle$ has the following ansatz:

$$
\left\langle O\left(k_{1}\right) O\left(k_{2}\right) J_{\mu}\left(k_{3}\right)\right\rangle=\left\langle O\left(k_{1}\right) O\left(k_{2}\right) j_{\mu}\left(k_{3}\right)\right\rangle-\frac{k_{3 \mu}}{k_{3}^{2}}\left(\left\langle O\left(k_{1}\right) O\left(-k_{1}\right)\right\rangle+\left\langle O\left(k_{2}\right) O\left(-k_{2}\right)\right\rangle\right)
$$


where $\langle O O j\rangle$ is the transverse part, which has the following ansatz

$$
\left\langle O\left(k_{1}\right) O\left(k_{2}\right) j_{\mu}\left(k_{3}\right)\right\rangle=A\left(k_{1}, k_{2}, k_{3}\right) k_{2}^{\nu} \pi_{\mu \nu}\left(k_{3}\right)
$$

The form factor $A$ is computed by solving the conformal ward identities, and is given by

$$
A\left(k_{1}, k_{2}, k_{3}\right)=2 I_{2\{111\}}
$$

We substitute this in the reconstruction formula (8.10) to get $\left\langle\mathrm{O}_{3} \mathrm{O}_{3} J\right\rangle$. Acting with $D_{12}^{++}$ on $\left\langle\mathrm{O}_{3} \mathrm{O}_{3} J\right\rangle$ gives:

$$
\begin{aligned}
\langle J J J\rangle & =D_{12}^{++}\left\langle O_{3} O_{3} J\right\rangle+\text { cyclic perms. } \\
& =D_{12}^{++}\left\langle O_{3} O_{3} j\right\rangle+\text { cyclic perms. }
\end{aligned}
$$

since $D_{12}^{++}$kills the longitudinal part. We now compute (8.13) to get the following explicit form of the form factor:

$$
\begin{aligned}
& C^{a b c}\left(k_{1}, k_{2}, k_{3}\right)= \\
& \begin{aligned}
=\frac{4 f^{a b c}}{J^{2}}[ & -\left(\left(k_{3} \cdot k_{1}\right)^{2}-k_{3} \cdot k_{1} k_{2}^{2}\right) I_{3\{101\}}+\left(\left(k_{2} \cdot k_{3}\right)^{2}+\left(k_{3} \cdot k_{1}\right)\left(k_{1}^{2}+k_{2}^{2}\right)\right) I_{3\{110\}} \\
& \left.+\left(-k_{2} \cdot k_{3} k_{1}^{2}+k_{2}^{2} k_{3}^{2}\right) I_{3\{011\}}\right]
\end{aligned}
\end{aligned}
$$

where $J^{2}=\left(k_{1}+k_{2}-k_{3}\right)\left(k_{1}-k_{2}+k_{3}\right)\left(-k_{1}+k_{2}+k_{3}\right)\left(k_{1}+k_{2}+k_{3}\right)$.

\section{Discussion}

In this paper we computed momentum space parity-odd 3-point functions of scalar operators and conserved currents in a CFT. We used conformal Ward identities to fix the parity-odd part of the $\langle J J O\rangle$ correlator. While using this method to fix more complicated 3-point functions of spinning operators such as $\langle J J J\rangle_{\text {odd }}$ or $\langle T T T\rangle_{\text {odd }}$, we faced technical difficulties arising from Schouten identities that relate various tensor structures. We leave this problem for a future work.

We defined parity-odd spin-raising and weight-shifting operators, and used them to compute $\langle J J J\rangle_{\text {odd }}$ and $\left\langle T T O_{\Delta}\right\rangle_{\text {odd }}$ for $\Delta=1,2$. However, we found it quite complicated to generalise this analysis to obtain correlators such as $\langle J J T\rangle_{\text {odd }}$ and $\langle T T T\rangle_{\text {odd }}$. The difficulty arises from the large number of possible paths to reach a specific correlator starting from a seed correlator. Another difficulty was the difference in the singularity structure of the seed correlator and the correlator of interest to us. We leave further investigation for the future.

Some of the directions that we would like to pursue in the near future are the following. It will be interesting to extend the analysis of $[16,27]$ and study the double copy structure of parity-odd correlation functions [89]. It will be interesting to generalise the construction of momentum space conformal blocks in [37] to the case where parity-odd contributions are important. In momentum space, conformal blocks are constructed quite simply by taking products of 3-point functions of primary operators (contrasted against position space, where an infinite sum over conformal descendants is required) [23, 26]. Recently, in 
the study of cosmological correlators [44, 47, 48], the form of tree-level four-point functions in momentum space was constrained. Tree level spinning correlators such as $\langle J J O O\rangle$ can get parity-odd contributions as they are built out of products of three-point functions. It would be interesting to find the explicit form of these and study their physical implications. Another interesting direction to pursue is to understand the parity-odd structure of 3-point correlators with operators of arbitrary spin. To do this, in addition to the techniques used in this paper, one could use the constraints imposed on the correlators by higher spin equations $[32,35]$. Solving higher spin equations requires us to include possible contact terms in the correlator. It would be interesting to classify both parity-even and parityodd contact terms for a given spinning correlator. One can also study momentum space correlation functions of spinning operators in supersymmetric theories [90-94].

\section{Acknowledgments}

We thank Nilay Kundu and Vinay Malvimat for discussions and collaboration at an early stage. The work of SJ and RRJ is supported by the Ramanujan Fellowship. AM would like to acknowledge the support of CSIR-UGC (JRF) fellowship (09/936(0212)/2019-EMR-I). The work of AS is supported by the KVPY scholarship. We acknowledge our debt to the people of India for their steady support of research in basic sciences.

\section{A Embedding space formalism}

In this section we briefly review some aspects of the embedding space formalism following [66]. Conformal invariance is most manifest in the embedding space formalism as the $d$-dimensional Euclidean conformal algebra is the $(d+2)$-dimensional Poincare algebra. The $d$-dimensional CFT correlator in position space can be written in terms of $(d+2)$ dimensional embedding space. The embeddding space coordinates $X^{M}$ are defined in terms of the position space coordinates $x^{i}$ as follows:

$$
\begin{aligned}
X^{A} & =\left(X^{+}, X^{-}, X^{i}\right)=\left(1, x^{2}, x^{i}\right) \\
Z^{B} & =\left(Z^{+}, Z^{-}, Z^{i}\right)=\left(0,2 z \cdot x, z^{i}\right) \\
X_{A} & =\left(X_{+}, X_{-}, X_{i}\right)=\left(x^{2} / 2,1 / 2,-x^{i}\right) \\
Z_{B} & =\left(Z_{+}, Z_{-}, Z_{i}\right)=\left(z \cdot x, 0,-z^{i}\right)
\end{aligned}
$$

where the $Z^{M}$ and $z^{i}$ are the null polarization vectors in the embedding space and position space, respectively. The derivative on the embedding space is defined as follows

$$
\frac{\partial}{\partial X^{M}}=-D \delta_{M}^{+}+P_{i} \delta_{M}^{i}
$$

where

$$
D=\Delta+x^{i} \frac{\partial}{\partial x^{i}}, \quad P_{i}=\frac{\partial}{\partial x^{i}}
$$


The $(d+2)$-dimensional space metric is as follows

$$
\eta_{M N}=\left(\begin{array}{ccccc}
0 & \frac{1}{2} & 0 & 0 & 0 \\
\frac{1}{2} & 0 & 0 & 0 & 0 \\
0 & 0 & -1 & 0 & 0 \\
0 & 0 & 0 & -1 & 0 \\
0 & 0 & 0 & 0 & -1
\end{array}\right)
$$

The embedding space coordinates are defined such that $Z \cdot X=X^{2}=Z^{2}=0$ because the CFT is defined on the null light cone of the AdS space.

The spin-raising and weight-shifting operators that we study in this paper are constructed to have two properties in the embedding space, namely transversality and interiority. Transversality requires that under the transformation $Z_{i} \rightarrow Z_{i}+\beta X_{i}$, the operators remain invariant, while under interiority, the operator must map null light cone to itself. The operators thus constructed are manifestly invariant under conformal transformations.

In the main text we have often used notations such as $\epsilon\left(Z_{1}, Z_{2}, X_{1}, X_{2}, X_{3}\right)$ :

$$
\epsilon\left(Z_{1}, Z_{2}, X_{1}, X_{2}, X_{3}\right)=\epsilon^{A B C D E} Z_{1 A} Z_{2 B} X_{1 C} X_{2 D} X_{3 E}
$$

\section{B Parity-odd two-point functions}

As is well known, scale invariance completely fixes CFT two-point functions. parity-odd structures can exist for two-point functions of spinning operators.

\section{B.1 Four and higher dimensions}

In four or higher dimensions, it is not possible to have any parity-odd two-point function of either spin-one or any other spinning symmetric spinning correlator. This is because a parity-odd correlator must necessarily involve the $\epsilon$ tensor and it is simple to show that it is impossible to have any parity-odd 2-point function of a spin-1 or any symmetric tensor operator.

\section{B.2 Three-dimensions}

In three-dimensions parity-odd two-point functions exist. These come from purely contact terms. ${ }^{11}$ We will look at the parity-odd 2-point functions of spin-one and spin two conserved currents.

$\left\langle\boldsymbol{J}^{\mu} \boldsymbol{J}^{\nu}\right\rangle_{\text {odd }}$. The general ansatz for the correlator is given by

$$
\left\langle J^{\mu}(k) J^{\nu}(-k)\right\rangle_{\text {odd }}=A(k) \epsilon^{\mu \nu k}
$$

The ansatz guarantees that the correlator is transverse to the momentum. Imposing scale invariance gives the following differential equation for the form factor $A(k)$ :

$$
k \frac{\partial}{\partial k} A(k)=0
$$

\footnotetext{
${ }^{11}$ In this case, the corresponding position space correlator with separated points vanishes.
} 
This implies that the form factor is just a constant in this case and we have:

$$
\left\langle J^{\mu}(k) J^{\nu}(-k)\right\rangle_{\text {odd }}=c_{J} \epsilon^{\mu \nu k}
$$

We will now consider the parity-odd 2-point function of the stress-tensor.

$\left\langle\boldsymbol{T}^{\mu \nu} \boldsymbol{T}^{\rho \sigma}\right\rangle_{\text {odd }}$. We consider the following ansatz for this correlator:

$$
\left\langle T^{\mu \nu}(k) T^{\rho \sigma}(-k)\right\rangle_{\text {odd }}=B(k) \Delta^{\mu \nu \rho \sigma}(k)
$$

where $\Delta^{\mu \nu \rho \sigma}(k)$ is a parity-odd, transverse-traceless projector given by:

$$
\Delta^{\mu \nu \rho \sigma}(k)=\epsilon^{\mu \rho k} \pi^{\nu \sigma}(k)+\epsilon^{\mu \sigma k} \pi^{\nu \rho}(k)+\epsilon^{\nu \sigma k} \pi^{\mu \rho}(k)+\epsilon^{\nu \rho k} \pi^{\mu \sigma}(k)
$$

where $\pi_{\nu}^{\mu}(k)$ is the same projector used in previous sections. The ansatz guarantees that the correlator is transverse and traceless.

The dilatation Ward identity gives the following equation for the form factor $B(k)$ :

$$
\left(k \frac{\partial}{\partial k}-2\right) B(k)=0
$$

This can be easily solved to get

$$
B(k)=c_{T} k^{2}
$$

Therefore, the correlator is given by

$$
\left\langle T^{\mu \nu}(k) T^{\rho \sigma}(-k)\right\rangle_{\text {odd }}=c_{T} \Delta^{\mu \nu \rho \sigma}(k) k^{2}
$$

\section{Schouten identities}

Here, we list the Schouten identities used in our calculations in the main text. The most general form of a Schouten identity in $d$-dimensions is

$$
\epsilon^{\left[\mu_{1} \mu_{2} \ldots \mu_{d}\right.} \delta^{\nu] \rho}=0
$$

In three-dimensions, this translates to

$$
\epsilon^{\mu_{1} \mu_{2} \mu_{3}} \delta^{\nu \rho}-\epsilon^{\mu_{2} \mu_{3} \nu} \delta^{\mu_{1} \rho}+\epsilon^{\mu_{3} \nu \mu_{1}} \delta^{\mu_{2} \rho}-\epsilon^{\nu \mu_{1} \mu_{2}} \delta^{\mu_{3} \rho}=0
$$

Dotting the indices in (C.2) with momenta lets us relate different epsilon structures that occur in the correlation functions calculated earlier. Dotting with $k_{1 \mu_{3}}, k_{1 \rho}$ and $k_{2 \nu}$ gives

$$
\epsilon^{\mu_{1} \mu_{2} k_{1}}\left(k_{1} \cdot k_{2}\right)+\epsilon^{\mu_{1} k_{1} k_{2}} k_{1}^{\mu_{2}}=\epsilon^{\mu_{1} \mu_{2} k_{2}} k_{1}^{2}+\epsilon^{\mu_{2} k_{1} k_{2}} k_{1}^{\mu_{1}}
$$

Similarly, dotting with $k_{1 \mu_{3}}, k_{2 \rho}$ and $k_{2 \nu}$

$$
\epsilon^{\mu_{1} \mu_{2} k_{2}}\left(k_{1} \cdot k_{2}\right)+\epsilon^{\mu_{2} k_{1} k_{2}} k_{2}^{\mu_{1}}=\epsilon^{\mu_{1} \mu_{2} k_{1}} k_{2}^{2}+\epsilon^{\mu_{1} k_{1} k_{2}} k_{2}^{\mu_{2}}
$$


Eqs. (C.3) and (C.4) were useful in rewriting the $\langle J J O\rangle$ ansatz. One can also derive these two identities by considering the contraction of three Levi-Civita tensors.

$$
\begin{aligned}
\epsilon^{\mu_{1} \alpha k_{1}} & \epsilon^{\beta k_{2} \mu_{2}} \epsilon_{\beta \rho \alpha}=\epsilon^{\mu_{1} \mu_{2} k_{1}} k_{2 \rho}+\epsilon^{\mu_{1} k_{1} k_{2}} \delta_{\rho}^{\mu_{2}} \\
\epsilon^{\beta k_{2} \mu_{2}} \epsilon^{\alpha k_{1} \mu_{1}} \epsilon_{\alpha \beta \rho} & =\epsilon^{\mu_{1} \mu_{2} k_{2}} k_{1 \rho}+\epsilon^{\mu_{2} k_{1} k_{2}} \delta_{\rho}^{\mu_{1}}
\end{aligned}
$$

Equating the r.h.s. of the two equations after dotting them with $k_{1}^{\rho}$ and $k_{2}^{\rho}$ respectively, we get back (C.3) and (C.4). Similarly, while checking the transverse identity for $\langle J J J\rangle$, we used the following Schouten identities

$$
\begin{aligned}
\epsilon^{k_{1} k_{2} \mu_{3}} k_{2}^{\mu_{2}} & =\epsilon^{k_{2} \mu_{3} \mu_{2}}\left(k_{1} \cdot k_{2}\right)+\epsilon^{\mu_{2} \mu_{3} k_{1}} k_{2}^{2}+\epsilon^{k_{1} k_{2} \mu_{2}} k_{2}^{\mu_{3}} \\
\epsilon^{k_{1} k_{2} \mu_{3}} k_{3}^{\mu_{2}} & =\epsilon^{k_{2} \mu_{3} \mu_{2}}\left(k_{1} \cdot k_{3}\right)+\epsilon^{\mu_{2} \mu_{3} k_{1}}\left(k_{2} \cdot k_{3}\right)+\epsilon^{\mu_{3} k_{1} k_{2}} k_{3}^{\mu_{3}}
\end{aligned}
$$

In four-dimensions, we use the following identities to rewrite the ansatz for $\langle J J J\rangle$.

$$
\begin{aligned}
& \left(k_{1} \cdot k_{2}\right) \epsilon_{\mu_{1} \mu_{2} \mu_{3} k_{1}}-k_{1 \mu_{1}} \epsilon_{k_{2} \mu_{2} \mu_{3} k_{1}}-k_{1 \mu_{2}} \epsilon_{\mu_{1} k_{2} \mu_{3} k_{1}}-k_{1 \mu_{3}} \epsilon_{\mu_{1} \mu_{2} k_{2} k_{1}}-k_{1}^{2} \epsilon_{\mu_{1} \mu_{2} \mu_{3} k_{2}}=0 \\
& \left(k_{1} \cdot k_{2}\right) \epsilon_{\mu_{1} \mu_{2} \mu_{3} k_{2}}-k_{2 \mu_{1}} \epsilon_{k_{2} \mu_{2} \mu_{3} k_{2}}-k_{2 \mu_{2}} \epsilon_{\mu_{1} k_{1} \mu_{3} k_{2}}-k_{2 \mu_{3}} \epsilon_{\mu_{1} \mu_{2} k_{1} k_{2}}-k_{2}^{2} \epsilon_{\mu_{1} \mu_{2} \mu_{3} k_{1}}=0
\end{aligned}
$$

\section{Details of $\left\langle\mathrm{TTO}_{2}\right\rangle$ computation}

In this section we give some details of the computation of the $\langle T T O\rangle$ correlator in the free fermion theory (7.5). We use the form of the stress tensor and the scalar operator as given in (7.8) and (7.9). The correlator of interest in terms of spinor fields is as follows:

$$
\begin{aligned}
& \left\langle T_{\mu \nu}\left(k_{1}\right) T_{\alpha \beta}\left(k_{2}\right) O\left(k_{3}\right)\right\rangle \\
& =\int_{123}\left\langle\bar{\psi}\left(l_{1}\right) \gamma_{\mu}\left(2 l_{1}-k_{1}\right)_{\nu} \psi\left(k_{1}-l_{1}\right) \bar{\psi}\left(l_{2}\right) \gamma_{\alpha}\left(2 l_{2}-k_{2}\right)_{\beta} \psi\left(k_{2}-l_{2}\right) \bar{\psi}\left(l_{3}\right) \psi\left(k_{3}-l_{3}\right)\right\rangle
\end{aligned}
$$

The Wick contraction $(1 \overline{3})(3 \overline{2})(2 \overline{1})$ gives: $^{12}$

$$
\int_{123} \operatorname{tr}\left(\gamma_{\mu} \gamma_{\rho} \gamma_{\sigma} \gamma_{\alpha} \gamma_{\tau}\right)\left(2 l_{1}-k_{1}\right)_{\nu}\left(2 l_{2}-k_{2}\right)_{\beta} \frac{l_{3}^{\rho} l_{2}^{\sigma} l_{1}^{\tau}}{l_{1}^{2} l_{2}^{2} l_{3}^{2}} \delta\left(k_{1}-l_{1}+l_{3}\right) \delta\left(k_{3}-l_{3}+l_{2}\right) \delta\left(k_{2}-l_{2}+l_{1}\right)
$$

where the fermion propagator given by:

$$
\left\langle\bar{\psi}_{\alpha}\left(k_{1}\right) \psi_{\beta}\left(k_{2}\right)\right\rangle=\delta^{(3)}\left(k_{1}+k_{2}\right) \frac{\not k_{1, \alpha \beta}}{k_{1}^{2}}
$$

was used. Computing the integrals over $l_{1}, l_{2}$ and using momentum conservation, we may write the integral (D.2) over a single variable as:

$$
\begin{aligned}
& \frac{1}{4}\left[\int_{3} \operatorname{tr}\left(\gamma_{\tau} \gamma_{\sigma} \gamma_{\alpha} \gamma_{\rho} \gamma_{\mu}\right)\left(2 l_{3}-k_{1}\right)_{\nu}\left(2 l_{3}+k_{2}\right)_{\beta} \frac{l_{3}^{\rho}\left(l_{3}-k_{1}\right)^{\tau}\left(l_{3}+k_{2}\right)^{\sigma}}{l_{3}^{2}\left(l_{3}+k_{2}\right)^{2}\left(l_{3}-k_{1}\right)^{2}}\right] \\
& + \text { symmetrize in }(\mu, \nu) \text { and }(\alpha, \beta)
\end{aligned}
$$

By projecting (D.4) with spin-2 projectors, one can now determine the transverse part of the correlator and hence, identify the form factors in (7.3) explicitly.

\footnotetext{
${ }^{12}$ We are not giving the details of the complex conjugate here as it gives the same result.
} 


\section{D.1 Transverse and trace Ward identities}

Here we provide an explicit verification of the transverse and trace Ward identities satisfied by $\langle T T O\rangle$. Contracting (D.4) with $k_{1 \mu}$ we obtain:

$$
\begin{aligned}
& \int_{3} \operatorname{tr}\left(\frac{1}{l_{3}-\not k_{1}} \frac{1}{l_{3}+\not k_{2}} \gamma_{\alpha} \frac{1}{l_{3}} \not k_{1}\right)\left(2 l_{3}-k_{1}\right)_{\nu}\left(2 l_{3}+k_{2}\right)_{\beta} \\
& +\int_{3} \operatorname{tr}\left(\frac{1}{\ell_{3}-\not k_{1}} \frac{1}{l_{3}+\not k_{2}} \gamma_{\alpha} \frac{1}{l_{3}} \gamma_{\mu}\right)\left(2 l_{3}-k_{1}\right) \cdot k_{1}\left(2 l_{3}+k_{2}\right)_{\beta} \\
& + \text { symmetrize in }(\alpha, \beta)
\end{aligned}
$$

which when simplified using:

$$
\begin{aligned}
\frac{1}{\not_{3}} \not k_{1} \frac{1}{l_{3}-\not k_{1}} & =\frac{1}{l_{3}-\not k_{1}}-\frac{1}{\not_{3}} \\
\left(2 l_{3}-k_{1}\right) \cdot k_{1} & =-\left(l_{3}-k_{1}\right)^{2}+l_{3}^{2}
\end{aligned}
$$

After a little algebra we get

$$
\frac{1}{128} k_{3}\left[k_{1 \beta}\left(\epsilon_{\alpha \nu k_{1}}-\epsilon_{\alpha \nu k_{2}}\right)+k_{1 \alpha}\left(\epsilon_{\beta \nu k_{1}}-\epsilon_{\beta \nu k_{2}}\right)-\delta_{\alpha, \nu} \epsilon_{\beta k_{1} k_{2}}-\delta_{\beta, \nu} \epsilon_{\alpha k_{1} k_{2}}\right]
$$

which precisely reproduces the transverse Ward identity given in (7.18). We now contract the $(\mu, \nu)$ indices to check the trace Ward identity:

$$
\begin{aligned}
& \int_{3} \operatorname{tr}\left(\frac{1}{\ell_{3}-\not k_{1}} \frac{1}{l_{3}+\not k_{2}} \gamma_{\alpha} \frac{1}{\not_{3}}\left(2 l_{3}-\not \ell_{1}\right)\right)\left(2 l_{3}+k_{2}\right)_{\beta} \\
& =\int_{3} \operatorname{tr}\left(\frac{1}{\not_{3}-\not l_{1}} \frac{1}{l_{3}+\not k_{2}} \gamma_{\alpha}\right)\left(2 l_{3}+k_{2}\right)_{\beta}+\int_{3} \operatorname{tr}\left(\frac{1}{l_{3}} \frac{1}{l_{3}+\not k_{2}} \gamma_{\alpha}\right)\left(2 l_{3}+k_{2}\right)_{\beta}
\end{aligned}
$$

In the first term, one may transform the integration variable to $l_{3} \rightarrow l_{3}-k_{2}$ :

$$
\begin{aligned}
& \int_{3} \operatorname{tr}\left(\frac{1}{l_{3}+\not k_{3}} \frac{1}{l_{3}} \gamma_{\alpha}\right)\left(2 l_{3}+k_{2}\right)_{\beta}+\int_{3} \operatorname{tr}\left(\frac{1}{l_{3}} \frac{1}{l_{3}+\not k_{2}} \gamma_{\alpha}\right)\left(2 l_{3}+k_{2}\right)_{\beta} \\
& =-2 i\left[\int_{3} \frac{\epsilon^{l_{3} k_{3} \alpha}}{l_{3}^{2}\left(l_{3}+k_{3}\right)^{2}}\left(2 l_{3}-k_{2}\right)_{\beta}-\int_{3} \frac{\epsilon_{3}^{l_{3} k_{2} \alpha}}{l_{3}^{2}\left(l_{3}+k_{2}\right)^{2}}\left(2 l_{3}+k_{2}\right)_{\beta}\right]
\end{aligned}
$$

Making use of the following identities

$$
\begin{aligned}
& \int_{l} \frac{l_{\mu}}{l^{2}(l+k)^{2}}=-\frac{k_{\mu}}{16 k} \\
& \int_{l} \frac{l_{\mu} l_{\nu}}{l^{2}(l+k)^{2}}=-\frac{k}{64} \eta_{\mu \nu}+\frac{3}{64 k} k_{\mu} k_{\nu}
\end{aligned}
$$

one can see that (D.9) vanishes and hence show that the correlator satisfies (7.17). 


\section{D.2 Details of longitudinal part}

In this section we compute the function derivative $\frac{\delta T_{\mu \nu}(x)}{\delta g_{\alpha \beta}(y)}$ in the free fermion theory, relevant to section 7.1. Since the functional dependence on the metric is only via the spin connection we have:

$$
\begin{aligned}
\left.\frac{\delta T_{\mu \nu}(x)}{\delta g_{\alpha \beta}(y)}\right|_{g_{\alpha \beta} \rightarrow \eta_{\alpha \beta}} & =\frac{1}{32}\left(\left.\frac{\delta \omega_{\nu}^{a b}(x)}{\delta g_{\alpha \beta}(y)}\right|_{g_{\alpha \beta} \rightarrow \eta_{\alpha \beta}} \bar{\psi}\left\{\gamma_{\mu}, \gamma_{a b}\right\} \psi+\left.\frac{\delta \omega_{\mu}^{a b}(x)}{\delta g_{\alpha \beta}(y)}\right|_{g_{\alpha \beta} \rightarrow \eta_{\alpha \beta}} \bar{\psi}\left\{\gamma_{\nu}, \gamma_{a b}\right\} \psi\right) \\
& =\frac{i}{16}\left(\frac{\delta \omega_{\nu}^{a b}(x)}{\delta g_{\alpha \beta}(y)} \epsilon_{a b \mu}+\frac{\delta \omega_{\mu}^{a b}(x)}{\delta g_{\alpha \beta}(y)} \epsilon_{a b \nu}\right) O(x)
\end{aligned}
$$

where

$$
\begin{aligned}
\left.\frac{\delta \omega_{\nu}^{a b}(x)}{\delta g_{\alpha \beta}(y)}\right|_{g_{\alpha \beta} \rightarrow \eta_{\alpha \beta}}= & \frac{\delta^{\sigma b} \delta^{a \tau}}{2} \\
& \times\left[\partial_{\sigma}\left(\delta_{(\tau}^{\alpha} \delta_{\nu)}^{\beta} \delta^{(3)}(x-y)\right)+\partial_{\nu}\left(\delta_{(\tau}^{\alpha} \delta_{\sigma)}^{\beta} \delta^{(3)}(x-y)\right)-\partial_{\tau}\left(\delta_{(\sigma}^{\alpha} \delta_{\nu)}^{\beta} \delta^{(3)}(x-y)\right)\right]
\end{aligned}
$$

The second line in (D.11) was obtained by recognising that in three-dimensions, $\gamma_{a b}=$ $\left[\sigma_{a}, \sigma_{b}\right]=2 i \epsilon_{a b c} \sigma^{c}$. In the limit, $g_{\alpha \beta} \rightarrow \eta_{\alpha \beta}$, the vierbiens go to $e_{\alpha}^{a} \rightarrow \delta_{\alpha}^{a}$ was also used. Simplifying the above expression to obtain,

$$
\frac{\delta \omega_{\nu}^{a b}(x)}{\delta g_{\alpha \beta}(y)} \epsilon_{a b \mu}=-\left[\epsilon_{\sigma \alpha \mu} \delta_{\beta \nu}+\epsilon_{\sigma \beta \mu} \delta_{\alpha \nu}\right] \partial^{\sigma} \delta^{(3)}(x-y)
$$

From here we get (7.12).

\section{E Parity-even spin-raising and weight-shifting operators}

In this section we list out all the parity-even weight-shifting operators used in the main text of the paper $[47,48]$.

The operator that decreases the scaling dimension of operators at points 1 and 2 is:

$$
W_{12}^{--}=\frac{1}{2} \vec{K}_{12}^{-} \cdot \vec{K}_{12}^{-}
$$

where

$$
K_{12}^{-\mu}=\partial_{k_{1 \mu}}-\partial_{k_{2 \mu}}
$$

We also use

$$
K_{12}^{+\mu}=\partial_{k_{1 \mu}}+\partial_{k_{2 \mu}}
$$

We can also define an operator that increases the scaling dimension at 2-points. Although this has a very complicated expression, it simplifies when acting on scalar operators and is given by:

$$
\begin{aligned}
W_{12}^{++}= & \left(k_{1} k_{2}\right)^{2} W_{12}^{--}-\left(d-2 \Delta_{1}\right)\left(d-2 \Delta_{2}\right) k_{1} \cdot k_{2} \\
& +\left(k_{2}^{2}\left(d-2 \Delta_{1}\right)\left(d-1-\Delta_{1}+k_{1} \cdot K_{12}\right)+(1 \leftrightarrow 2)\right)
\end{aligned}
$$


$D_{11}$ raises the spin of the operator at point 1 and simultaneously lowers its weight. This was used in the construction of both $\langle T T O\rangle$ and $\langle J J J\rangle$ :

$$
D_{11}=\left(\Delta_{2}-3+\vec{k}_{2} \cdot \vec{K}_{12}\right) \vec{z}_{1} \cdot \vec{K}_{12}-\left(\vec{k}_{2} \cdot \vec{z}_{1}\right) W_{12}^{--}-\left(\vec{z}_{2} \cdot \vec{K}_{12}\right)\left(\vec{z}_{1} \cdot \partial_{\vec{z}_{2}}\right)+\left(\vec{z}_{1} \cdot \vec{z}_{2}\right) \partial_{\vec{z}_{2}} \cdot \vec{K}_{12}
$$

We can similarly define $D_{22}$ and $D_{33}$ by doing cyclic permutations of the momenta and polarization vectors in (E.5). For example,

$$
D_{22}\left(\left(k_{1}, z_{1}\right),\left(k_{2}, z_{2}\right),\left(k_{3}, z_{3}\right)\right)=D_{11}\left(\left(k_{3}, z_{3}\right),\left(k_{1}, z_{1}\right),\left(k_{2}, z_{2}\right)\right)
$$

$S_{12}^{++}$raises the spin at points 1 and 2 :

$$
\begin{aligned}
S_{12}^{++}= & \left(s_{1}+\Delta_{1}-1\right)\left(s_{2}+\Delta_{2}-1\right) z_{1} \cdot z_{2}-\left(z_{1} \cdot k_{1}\right)\left(z_{2} \cdot k_{2}\right) W_{12}^{--} \\
& +\left[\left(s_{1}+\Delta_{1}-1\right)\left(k_{2} \cdot z_{2}\right)\left(z_{1} \cdot K_{12}\right)+(1 \leftrightarrow 2)\right]
\end{aligned}
$$

$S_{23}^{++}$and $S_{13}^{++}$are once again defined by cyclic permutations of (E.7).

The operator $H_{12}$ which raises the spin at points 1 and 2 and also lowers the weight at both the points is given by:

$$
H_{12}=2\left(z_{1} \cdot K_{12}\right)\left(z_{2} \cdot K_{12}\right)-2\left(z_{1} \cdot z_{2}\right) W_{12}^{--}
$$

The operator that raises the spin at point 1 and simulataneously lowers the weight at point 2 is given by:

$$
D_{12}=\left(\Delta_{1}+s_{1}-1\right) z_{1} \cdot K_{12}-\left(z_{1} \cdot k_{1}\right) W_{12}^{--}
$$

A $(1 \leftrightarrow 2)$ exchange in this operator gives $D_{21}$. Both of these were used in the construction of $\langle T T O\rangle$.

\section{F Embedding space parity-odd correlation functions in four-dimensions}

In this appendix, we show that $\langle J J T\rangle_{\text {odd }}$ and $\langle T T T\rangle_{\text {odd }}$ are zero in four-dimensions.

\section{F.1 $\langle J J T\rangle_{\text {odd }}$}

We first write the $\langle J J T\rangle_{\text {odd }}$ correlator in a basis of (embedding space) conformally invariant structures (the notation used is that of [66])

$$
\left\langle J\left(Z_{1}, X_{1}\right) J\left(Z_{2}, X_{2}\right) T\left(Z_{3}, X_{3}\right)\right\rangle_{\text {odd }}=c_{1} \epsilon\left(Z_{1} Z_{2} Z_{3} X_{1} X_{2} X_{3}\right) \frac{V_{3}}{X_{12}^{3 / 2} X_{23}^{5 / 2} X_{31}^{5 / 2}}
$$

Under simultaneous exchange of $\left(X_{1}, X_{2}\right)$ and $\left(Z_{1}, Z_{2}\right)$ we have

$$
V_{3} \rightarrow-V_{3}
$$

Hence, symmetry consideration demands that $c_{1}=0$. This implies that the parity-odd $\langle J J T\rangle$ correlator vanishes in four-dimensions. In momentum space, it can be a contact term. 


\section{F.2 $\langle T T T\rangle_{\text {odd }}$}

We first write the correlator in a basis of conformally invariant structures

$$
\begin{aligned}
& \left\langle T\left(Z_{1}, X_{1}\right) T\left(Z_{2}, X_{2}\right) T\left(Z_{3}, X_{3}\right)\right\rangle_{\text {odd }} \\
& =\epsilon\left(Z_{1} Z_{2} Z_{3} X_{1} X_{2} X_{3}\right) \sum_{n_{12}, n_{13}, n_{23}} A_{n_{12}, n_{13}, n_{23}} \frac{V_{1}^{1-n_{12}-n_{13}} V_{2}^{1-n_{12}-n_{23}} V_{3}^{1-n_{13}-n_{23}} H_{12}^{n_{12}} H_{13}^{n_{13}} H_{23}^{n_{23}}}{X_{12}^{7 / 2} X_{13}^{7 / 2} X_{23}^{7 / 2}} \\
& =\epsilon\left(Z_{1} Z_{2} Z_{3} X_{1} X_{2} X_{3}\right) \\
& \times\left[A_{000} \frac{V_{1} V_{2} V_{3}}{X_{12}^{7 / 2} X_{13}^{7 / 2} X_{23}^{7 / 2}}+A_{001} \frac{V_{2} H_{13}}{X_{12}^{7 / 2} X_{13}^{7 / 2} X_{23}^{7 / 2}}+A_{010} \frac{V_{1} H_{23}}{X_{12}^{7 / 2} X_{13}^{7 / 2} X_{23}^{7 / 2}}+A_{100} \frac{V_{3} H_{12}}{X_{12}^{7 / 2} X_{13}^{7 / 2} X_{23}^{7 / 2}}\right]
\end{aligned}
$$

Under simultaneous $Z_{1} \leftrightarrow Z_{2}, X_{1} \leftrightarrow X_{2}$ exchange we have

$$
\begin{aligned}
& V_{1} \rightarrow-V_{2}, \quad V_{3} \rightarrow-V_{3}, \quad H_{12} \rightarrow H_{12}, \quad H_{13} \rightarrow H_{23} \\
& \left\langle T\left(Z_{1}, X_{1}\right) T\left(Z_{2}, X_{2}\right) T\left(Z_{3}, X_{3}\right)\right\rangle_{\text {odd }} \\
& =-\epsilon\left(Z_{1} Z_{2} Z_{3} X_{1} X_{2} X_{3}\right) \\
& \times\left[A_{000} \frac{V_{1} V_{2} V_{3}}{X_{12}^{7 / 2} X_{13}^{7 / 2} X_{23}^{7 / 2}}+A_{001} \frac{V_{1} H_{23}}{X_{12}^{7 / 2} X_{13}^{7 / 2} X_{23}^{7 / 2}}+A_{010} \frac{V_{2} H_{13}}{X_{12}^{7 / 2} X_{13}^{7 / 2} X_{23}^{7 / 2}}+A_{100} \frac{V_{3} H_{12}}{X_{12}^{7 / 2} X_{13}^{7 / 2} X_{23}^{7 / 2}}\right]
\end{aligned}
$$

Therefore, we must have

$$
A_{000}=0, \quad A_{001}=-A_{010}, \quad A_{100}=0
$$

Hence,

$$
\begin{aligned}
\left\langle T\left(Z_{1}, X_{1}\right) T\left(Z_{2}, X_{2}\right) T\left(Z_{3}, X_{3}\right)\right\rangle_{\text {odd }}= & A_{001} \epsilon\left(Z_{1} Z_{2} Z_{3} X_{1} X_{2} X_{3}\right) \\
& \times\left[\frac{V_{1} H_{23}}{X_{12}^{7 / 2} X_{13}^{7 / 2} X_{23}^{7 / 2}}-\frac{V_{2} H_{13}}{X_{12}^{7 / 2} X_{13}^{7 / 2} X_{23}^{7 / 2}}\right]
\end{aligned}
$$

Now, under simultaneous exchange of $\left(Z_{2}, Z_{3}\right)$ and $\left(X_{2}, X_{3}\right)$

$$
V_{1} \rightarrow-V_{1} \quad V_{2} \rightarrow-V_{3} \quad H_{23} \rightarrow H_{23} \quad H_{13} \rightarrow H_{12}
$$

Therefore,

$$
\begin{aligned}
\left\langle T\left(Z_{1}, X_{1}\right) T\left(Z_{2}, X_{2}\right) T\left(Z_{3}, X_{3}\right)\right\rangle_{\text {odd }}= & A_{001} \epsilon\left(Z_{1} Z_{2} Z_{3} X_{1} X_{2} X_{3}\right) \\
& \times\left[-\frac{V_{1} H_{23}}{X_{12}^{7 / 2} X_{13}^{7 / 2} X_{23}^{7 / 2}}+\frac{V_{3} H_{12}}{X_{12}^{7 / 2} X_{13}^{7 / 2} X_{23}^{7 / 2}}\right]
\end{aligned}
$$

Hence, we must have $A_{001}=0$. Therefore, symmetry considerations force $\langle T T T\rangle_{\text {odd }}$ to be zero.

Open Access. This article is distributed under the terms of the Creative Commons Attribution License (CC-BY 4.0), which permits any use, distribution and reproduction in any medium, provided the original author(s) and source are credited. 


\section{References}

[1] C. Corianò, L. Delle Rose, E. Mottola and M. Serino, Solving the conformal constraints for scalar operators in momentum space and the evaluation of Feynman's master integrals, JHEP 07 (2013) 011 [arXiv: 1304.6944] [INSPIRE].

[2] A. Bzowski, P. McFadden and K. Skenderis, Implications of conformal invariance in momentum space, JHEP 03 (2014) 111 [arXiv:1304.7760] [INSPIRE].

[3] L. Bonora, A.D. Pereira and B. Lima de Souza, Regularization of energy-momentum tensor correlators and parity-odd terms, JHEP 06 (2015) 024 [arXiv: 1503.03326] [INSPIRE].

[4] A. Bzowski, P. McFadden and K. Skenderis, Scalar 3-point functions in CFT: renormalisation, $\beta$-functions and anomalies, JHEP 03 (2016) 066 [arXiv:1510.08442] [INSPIRE].

[5] A. Bzowski, P. McFadden and K. Skenderis, Evaluation of conformal integrals, JHEP 02 (2016) 068 [arXiv:1511.02357] [INSPIRE].

[6] L. Bonora and B. Lima de Souza, Pure contact term correlators in CFT, Bled Workshops Phys. 16 (2015) 22 [arXiv:1511.06635] [InSPIRE].

[7] L. Bonora, M. Cvitan, P. Dominis Prester, B. Lima de Souza and I. Smolić, Massive fermion model in 3d and higher spin currents, JHEP 05 (2016) 072 [arXiv:1602.07178] [INSPIRE].

[8] B. Lima de Souza, CFT's, contact terms and anomalies, Ph.D. thesis, SISSA, Trieste, Italy (2016) [INSPIRE].

[9] A. Bzowski, P. McFadden and K. Skenderis, Renormalised 3-point functions of stress tensors and conserved currents in CFT, JHEP 11 (2018) 153 [arXiv:1711.09105] [INSPIRE].

[10] C. Corianò and M.M. Maglio, Exact correlators from conformal Ward identities in momentum space and the perturbative TJJ vertex, Nucl. Phys. B 938 (2019) 440 [arXiv: 1802.07675] [INSPIRE].

[11] H. Isono, T. Noumi and G. Shiu, Momentum space approach to crossing symmetric CFT correlators, JHEP 07 (2018) 136 [arXiv: 1805.11107] [INSPIRE].

[12] A. Bzowski, P. McFadden and K. Skenderis, Renormalised CFT 3-point functions of scalars, currents and stress tensors, JHEP 11 (2018) 159 [arXiv: 1805.12100] [INSPIRE].

[13] M. Gillioz, Momentum-space conformal blocks on the light cone, JHEP 10 (2018) 125 [arXiv: 1807.07003] [INSPIRE].

[14] C. Corianò and M.M. Maglio, Conformal Ward identities and the coupling of QED and QCD to gravity, EPJ Web Conf. 192 (2018) 00047 [arXiv:1809.05940] [INSPIRE].

[15] S. Albayrak and S. Kharel, Towards the higher point holographic momentum space amplitudes, JHEP 02 (2019) 040 [arXiv: 1810.12459] [INSPIRE].

[16] J.A. Farrow, A.E. Lipstein and P. McFadden, Double copy structure of CFT correlators, JHEP 02 (2019) 130 [arXiv: 1812.11129] [INSPIRE].

[17] H. Isono, T. Noumi and T. Takeuchi, Momentum space conformal three-point functions of conserved currents and a general spinning operator, JHEP 05 (2019) 057 [arXiv:1903.01110] [INSPIRE]. 
[18] C. Corianò and M.M. Maglio, On some hypergeometric solutions of the conformal Ward identities of scalar 4-point functions in momentum space, JHEP 09 (2019) 107 [arXiv: 1903.05047] [INSPIRE].

[19] S. Albayrak, C. Chowdhury and S. Kharel, New relation for Witten diagrams, JHEP 10 (2019) 274 [arXiv: 1904.10043] [InSPIRE].

[20] S. Albayrak and S. Kharel, Towards the higher point holographic momentum space amplitudes. Part II. Gravitons, JHEP 12 (2019) 135 [arXiv: 1908.01835] [INSPIRE].

[21] H. Isono, T. Noumi and G. Shiu, Momentum space approach to crossing symmetric CFT correlators. Part II. General spacetime dimension, JHEP 10 (2019) 183 [arXiv:1908.04572] [INSPIRE].

[22] T. Bautista and H. Godazgar, Lorentzian CFT 3-point functions in momentum space, JHEP 01 (2020) 142 [arXiv: 1908.04733] [INSPIRE].

[23] M. Gillioz, Conformal 3-point functions and the Lorentzian OPE in momentum space, Commun. Math. Phys. 379 (2020) 227 [arXiv: 1909.00878] [INSPIRE].

[24] A. Bzowski, P. McFadden and K. Skenderis, Conformal n-point functions in momentum space, Phys. Rev. Lett. 124 (2020) 131602 [arXiv:1910.10162] [INSPIRE].

[25] C. Corianò, M.M. Maglio and D. Theofilopoulos, Four-point functions in momentum space: conformal Ward identities in the scalar/tensor case, Eur. Phys. J. C 80 (2020) 540 [arXiv: 1912.01907] [INSPIRE].

[26] M. Gillioz, X. Lu, M.A. Luty and G. Mikaberidze, Convergent momentum-space OPE and bootstrap equations in conformal field theory, JHEP 03 (2020) 102 [arXiv:1912.05550] [INSPIRE].

[27] A.E. Lipstein and P. McFadden, Double copy structure and the flat space limit of conformal correlators in even dimensions, Phys. Rev. D 101 (2020) 125006 [arXiv:1912.10046] [INSPIRE].

[28] S. Albayrak, C. Chowdhury and S. Kharel, Study of momentum space scalar amplitudes in AdS spacetime, Phys. Rev. D 101 (2020) 124043 [arXiv:2001.06777] [InSPIRE].

[29] C. Corianò and M.M. Maglio, The generalized hypergeometric structure of the Ward identities of CFT's in momentum space in $d>2$, Axioms 9 (2020) 54 [arXiv: 2001.09622] [INSPIRE].

[30] M. Gillioz, M. Meineri and J. Penedones, A scattering amplitude in conformal field theory, JHEP 11 (2020) 139 [arXiv:2003.07361] [INSPIRE].

[31] M. Serino, The four-point correlation function of the energy-momentum tensor in the free conformal field theory of a scalar field, Eur. Phys. J. C 80 (2020) 686 [arXiv:2004.08668] [INSPIRE].

[32] S. Jain, R.R. John and V. Malvimat, Momentum space spinning correlators and higher spin equations in three dimensions, JHEP 11 (2020) 049 [arXiv: 2005. 07212] [INSPIRE].

[33] S. Albayrak and S. Kharel, Spinning loop amplitudes in anti-de Sitter space, Phys. Rev. D 103 (2021) 026004 [arXiv:2006.12540] [INSPIRE].

[34] A. Bzowski, P. McFadden and K. Skenderis, Conformal correlators as simplex integrals in momentum space, JHEP 01 (2021) 192 [arXiv:2008.07543] [INSPIRE].

[35] S. Jain, R.R. John and V. Malvimat, Constraining momentum space correlators using slightly broken higher spin symmetry, JHEP 04 (2021) 231 [arXiv: 2008.08610] [INSPIRE]. 
[36] C. Armstrong, A.E. Lipstein and J. Mei, Color/kinematics duality in AdS 4 , JHEP 02 (2021) 194 [arXiv: 2012.02059] [INSPIRE].

[37] M. Gillioz, Conformal partial waves in momentum space, SciPost Phys. 10 (2021) 081 [arXiv: 2012.09825] [INSPIRE].

[38] S. Albayrak, S. Kharel and D. Meltzer, On duality of color and kinematics in (A)dS momentum space, JHEP 03 (2021) 249 [arXiv:2012.10460] [INSPIRE].

[39] J.M. Maldacena and G.L. Pimentel, On graviton non-Gaussianities during inflation, JHEP 09 (2011) 045 [arXiv: 1104.2846] [inSPIRE].

[40] I. Mata, S. Raju and S. Trivedi, CMB from CFT, JHEP 07 (2013) 015 [arXiv:1211.5482] [INSPIRE].

[41] A. Ghosh, N. Kundu, S. Raju and S.P. Trivedi, Conformal invariance and the four point scalar correlator in slow-roll inflation, JHEP 07 (2014) 011 [arXiv:1401.1426] [INSPIRE].

[42] N. Kundu, A. Shukla and S.P. Trivedi, Constraints from conformal symmetry on the three point scalar correlator in inflation, JHEP 04 (2015) 061 [arXiv:1410.2606] [INSPIRE].

[43] N. Arkani-Hamed and J. Maldacena, Cosmological collider physics, arXiv:1503.08043 [INSPIRE].

[44] N. Arkani-Hamed, D. Baumann, H. Lee and G.L. Pimentel, The cosmological bootstrap: inflationary correlators from symmetries and singularities, JHEP 04 (2020) 105 [arXiv: 1811.00024] [INSPIRE].

[45] C. Sleight, A Mellin space approach to cosmological correlators, JHEP 01 (2020) 090 [arXiv: 1906.12302] [INSPIRE].

[46] C. Sleight and M. Taronna, Bootstrapping inflationary correlators in Mellin space, JHEP 02 (2020) 098 [arXiv: 1907.01143] [INSPIRE].

[47] D. Baumann, C. Duaso Pueyo, A. Joyce, H. Lee and G.L. Pimentel, The cosmological bootstrap: weight-shifting operators and scalar seeds, JHEP 12 (2020) 204 [arXiv: 1910.14051] [INSPIRE].

[48] D. Baumann, C. Duaso Pueyo, A. Joyce, H. Lee and G.L. Pimentel, The cosmological bootstrap: spinning correlators from symmetries and factorization, arXiv:2005.04234 [INSPIRE].

[49] Y. Huh, P. Strack and S. Sachdev, Conserved current correlators of conformal field theories in $2+1$ dimensions, Phys. Rev. B 88 (2013) 155109 [Erratum ibid. 90 (2014) 199902] [arXiv:1307.6863] [INSPIRE].

[50] D. Chowdhury, S. Raju, S. Sachdev, A. Singh and P. Strack, Multipoint correlators of conformal field theories: implications for quantum critical transport, Phys. Rev. B $\mathbf{8 7}$ (2013) 085138 [arXiv: 1210 .5247] [INSPIRE].

[51] M. Gillioz, X. Lu and M.A. Luty, Scale anomalies, states, and rates in conformal field theory, JHEP 04 (2017) 171 [arXiv: 1612.07800] [INSPIRE].

[52] C. Corianò, M.M. Maglio and E. Mottola, TTT in CFT: trace identities and the conformal anomaly effective action, Nucl. Phys. B 942 (2019) 303 [arXiv:1703.08860] [INSPIRE].

[53] M. Gillioz, X. Lu and M.A. Luty, Graviton scattering and a sum rule for the $c$ anomaly in $4 D$ CFT, JHEP 09 (2018) 025 [arXiv: 1801.05807] [INSPIRE]. 
[54] C. Corianò and M.M. Maglio, Renormalization, conformal Ward identities and the origin of a conformal anomaly pole, Phys. Lett. B 781 (2018) 283 [arXiv:1802.01501] [INSPIRE].

[55] C. Corianò and M.M. Maglio, Conformal field theory in momentum space and anomaly actions in gravity: the analysis of 3- and 4-point functions, arXiv:2005.06873 [INSPIRE].

[56] N. Anand, Z.U. Khandker and M.T. Walters, Momentum space CFT correlators for Hamiltonian truncation, JHEP 10 (2020) 095 [arXiv: 1911.02573] [INSPIRE].

[57] E. Katz, Z.U. Khandker and M.T. Walters, A conformal truncation framework for infinite-volume dynamics, JHEP 07 (2016) 140 [arXiv:1604.01766] [INSPIRE].

[58] A.M. Polyakov, Nonhamiltonian approach to conformal quantum field theory, Zh. Eksp. Teor. Fiz. 66 (1974) 23 [Sov. Phys. JETP 39 (1974) 9] [INSPIRE].

[59] S. Raju, New recursion relations and a flat space limit for AdS/CFT correlators, Phys. Rev. D 85 (2012) 126009 [arXiv:1201.6449] [INSPIRE].

[60] J. Penedones, Writing CFT correlation functions as AdS scattering amplitudes, JHEP 03 (2011) 025 [arXiv:1011.1485] [INSPIRE].

[61] A.L. Fitzpatrick and J. Kaplan, Analyticity and the holographic S-matrix, JHEP 10 (2012) 127 [arXiv: 1111.6972] [inSPIRE].

[62] M. Gary, S.B. Giddings and J. Penedones, Local bulk S-matrix elements and CFT singularities, Phys. Rev. D 80 (2009) 085005 [arXiv:0903.4437] [InSPIRE].

[63] M. Gary and S.B. Giddings, The flat space S-matrix from the AdS/CFT correspondence?, Phys. Rev. D 80 (2009) 046008 [arXiv: 0904.3544] [INSPIRE].

[64] S. Komatsu, M.F. Paulos, B.C. Van Rees and X. Zhao, Landau diagrams in AdS and S-matrices from conformal correlators, JHEP 11 (2020) 046 [arXiv: 2007.13745] [INSPIRE].

[65] S. Giombi, S. Prakash and X. Yin, A note on CFT correlators in three dimensions, JHEP 07 (2013) 105 [arXiv: 1104.4317] [INSPIRE].

[66] M.S. Costa, J. Penedones, D. Poland and S. Rychkov, Spinning conformal correlators, JHEP 11 (2011) 071 [arXiv: 1107.3554] [InSPIRE].

[67] M.S. Costa, J. Penedones, D. Poland and S. Rychkov, Spinning conformal blocks, JHEP 11 (2011) 154 [arXiv:1109.6321] [INSPIRE].

[68] S.D. Chowdhury, J.R. David and S. Prakash, Spectral sum rules for conformal field theories in arbitrary dimensions, JHEP 07 (2017) 119 [arXiv: 1612.00609] [INSPIRE].

[69] S.D. Chowdhury, J.R. David and S. Prakash, Constraints on parity violating conformal field theories in $d=3$, JHEP 11 (2017) 171 [arXiv:1707.03007] [INSPIRE].

[70] S.D. Chowdhury, J.R. David and S. Prakash, Bootstrap and collider physics of parity violating conformal field theories in $d=3$, JHEP 04 (2019) 023 [arXiv: 1812.07774] [INSPIRE].

[71] O. Aharony, G. Gur-Ari and R. Yacoby, $d=3$ bosonic vector models coupled to Chern-Simons gauge theories, JHEP 03 (2012) 037 [arXiv:1110.4382] [INSPIRE].

[72] S. Giombi, S. Minwalla, S. Prakash, S.P. Trivedi, S.R. Wadia and X. Yin, Chern-Simons theory with vector fermion matter, Eur. Phys. J. C 72 (2012) 2112 [arXiv:1110.4386] [INSPIRE]. 
[73] J. Maldacena and A. Zhiboedov, Constraining conformal field theories with a slightly broken higher spin symmetry, Class. Quant. Grav. 30 (2013) 104003 [arXiv:1204.3882] [InSPIRE].

[74] O. Aharony, G. Gur-Ari and R. Yacoby, Correlation functions of large $N$ Chern-Simons-matter theories and bosonization in three dimensions, JHEP 12 (2012) 028 [arXiv: 1207.4593] [INSPIRE].

[75] G. Gur-Ari and R. Yacoby, Correlators of large $N$ fermionic Chern-Simons vector models, JHEP 02 (2013) 150 [arXiv:1211.1866] [InSPIRE].

[76] S. Giombi, V. Gurucharan, V. Kirilin, S. Prakash and E. Skvortsov, On the higher-spin spectrum in large N Chern-Simons vector models, JHEP 01 (2017) 058 [arXiv:1610.08472] [INSPIRE].

[77] V. Guru Charan and S. Prakash, On the higher spin spectrum of Chern-Simons theory coupled to fermions in the large flavour limit, JHEP 02 (2018) 094 [arXiv:1711.11300] [INSPIRE].

[78] O. Aharony, S. Jain and S. Minwalla, Flows, fixed points and duality in Chern-Simons-matter theories, JHEP 12 (2018) 058 [arXiv:1808.03317] [INSPIRE].

[79] E. Skvortsov, Light-front bootstrap for Chern-Simons matter theories, JHEP 06 (2019) 058 [arXiv: 1811.12333] [INSPIRE].

[80] R.R. Kalloor, Four-point functions in large N Chern-Simons fermionic theories, JHEP 10 (2020) 028 [arXiv : 1910.14617] [INSPIRE].

[81] H. Osborn and A.C. Petkou, Implications of conformal invariance in field theories for general dimensions, Annals Phys. 231 (1994) 311 [hep-th/9307010] [INSPIRE].

[82] J. Soda, H. Kodama and M. Nozawa, Parity violation in graviton non-Gaussianity, JHEP 08 (2011) 067 [arXiv: 1106.3228] [INSPIRE].

[83] A. Lue, L.-M. Wang and M. Kamionkowski, Cosmological signature of new parity violating interactions, Phys. Rev. Lett. 83 (1999) 1506 [astro-ph/9812088] [INSPIRE].

[84] M. Shiraishi, M. Liguori and J.R. Fergusson, General parity-odd CMB bispectrum estimation, JCAP 05 (2014) 008 [arXiv: 1403.4222] [INSPIRE].

[85] M. Shiraishi, M. Liguori and J.R. Fergusson, Observed parity-odd CMB temperature bispectrum, JCAP 01 (2015) 007 [arXiv: 1409. 0265] [INSPIRE].

[86] D. Karateev, P. Kravchuk and D. Simmons-Duffin, Weight shifting operators and conformal blocks, JHEP 02 (2018) 081 [arXiv: 1706. 07813] [INSPIRE].

[87] A. Bzowski, TripleK: a Mathematica package for evaluating triple-K integrals and conformal correlation functions, Comput. Phys. Commun. 258 (2021) 107538 [arXiv:2005.10841] [INSPIRE].

[88] V.K. Dobrev, V.B. Petkova, S.G. Petrova and I.T. Todorov, Dynamical derivation of vacuum operator product expansion in Euclidean conformal quantum field theory, Phys. Rev. D 13 (1976) 887 [INSPIRE].

[89] S. Jain, R.R. John, A. Mehta, A.A. Nizami and A. Suresh, Double copy structure of parity-violating CFT correlators, JHEP 07 (2021) 033 [arXiv:2104.12803] [INSPIRE].

[90] E.I. Buchbinder, S.M. Kuzenko and I.B. Samsonov, Superconformal field theory in three dimensions: correlation functions of conserved currents, JHEP 06 (2015) 138 [arXiv: 1503.04961] [INSPIRE]. 
[91] E.I. Buchbinder, S.M. Kuzenko and I.B. Samsonov, Implications of $N=4$ superconformal symmetry in three spacetime dimensions, JHEP 08 (2015) 125 [arXiv:1507.00221] [INSPIRE].

[92] S.M. Kuzenko and I.B. Samsonov, Implications of $N=5,6$ superconformal symmetry in three spacetime dimensions, JHEP 08 (2016) 084 [arXiv: 1605.08208] [INSPIRE].

[93] O. Aharony and A. Sharon, Large $N$ renormalization group flows in $3 d N=1$ Chern-Simons-matter theories, JHEP 07 (2019) 160 [arXiv:1905.07146] [INSPIRE].

[94] K. Inbasekar, S. Jain, V. Malvimat, A. Mehta, P. Nayak and T. Sharma, Correlation functions in $N=2$ supersymmetric vector matter Chern-Simons theory, JHEP 04 (2020) 207 [arXiv: 1907.11722] [INSPIRE]. 